\title{
Functional Properties of the MAP Kinase UeKpp2 in Ustilago esculenta
}

\author{
Yafen Zhang ${ }^{\dagger}$, Yingli Hut, Qianchao Cao, Yumei Yin, Wenqiang Xia, Haifeng Cui, \\ Xiaoping Yu and Zihong Ye*
}

Zhejiang Provincial Key Laboratory of Biometrology and Inspection \& Quarantine, College of Life Sciences, China Jiliang University, Hangzhou, China

OPEN ACCESS

Edited by:

Zuhua He,

Center for Excellence in Molecular, Plant Sciences (CAS), China

Reviewed by:

Zejian GUO,

China Agricultural University, China

Dong-Lei Yang,

Nanjing Agricultural University, China

${ }^{*}$ Correspondence:

Zihong Ye

13656686088@163.com zhye@cjlu.edu.cn

tThese authors have contributed equally to this work

Specialty section:

This article was submitted to

Plant Microbe Interactions,

a section of the journal

Frontiers in Microbiology

Received: 02 January 2020

Accepted: 28 April 2020

Published: 09 June 2020

Citation:

Zhang Y, Hu Y, Cao Q, Yin Y, Xia W, Cui H, Yu X and Ye Z (2020) Functional Properties of the MAP Kinase UeKpp2 in Ustilago esculenta.

Front. Microbiol. 11:1053.

doi: 10.3389/fmicb.2020.01053
Ustilago esculenta undergoes an endophytic life cycle in Zizania latifolia. It induces the stem of its host to swell, forming the edible galls called jiaobai in China, which are the second most commonly cultivated aquatic vegetable in China. Z. latifolia raised for jiaobai can only reproduce asexually because the $U$. esculenta infection completely inhibits flowering. The infection and proliferation in the host plants during the formation of edible gall differ from those of conventional pathogens. Previous studies have shown a close relationship between mitogen-activated protein kinase (MAPK) and fungal pathogenesis. In this study, we explored the functional properties of the MAPK UeKpp2. Cross-species complementation assays were carried out, which indicated a functional complementation between the UeKpp2 of $U$. esculenta and the Kpp2 of Ustilago maydis. Next, UeKpp2 mutants of the UeT14 and the UeT55 sporidia background were generated; these showed an aberrant morphology of budding cells, and attenuated mating and filamentous growth in vitro, in the context of normal pathogenicity. Interestingly, we identified another protein kinase, UeUkc1, which acted downstream of UeKpp2 and may participate in the regulation of cell shape. We also found a defect of filamentous growth in UeKpp2 mutants that was not related to a defect of the induction of mating-type genes but was directly related to a defect in UeRbf1 induction. Overall, our results indicate an important role for UeKpp2 in U. esculenta that is slightly different from those reported for other smut fungi.

Keywords: Ustilago esculenta, UeKpp2, mating, filamentous growth, UeRbf1, UeUkc1

\section{INTRODUCTION}

The smut fungus Ustilago esculenta induces a swollen stem in Zizania latifolia, its only known host (Chung and Tzeng, 2004). Z. latifolia individuals colonized with U. esculenta are widely cultivated in East and Southeast Asia and are the second most commonly cultivated aquatic vegetable grown in China, where they are called jiaobai because their swollen stems are delicious and nutritious (Suzuki et al., 2012; Jiang et al., 2016; Jose et al., 2016; Yan et al., 2018). Due to the fungal infection, jiaobai cannot flower or produce seeds (Guo et al., 2015). It reproduces asexually. It is now generally accepted that there are two type strains of U. esculenta, T and MT. The T type strain shows the pathogenic life cycle of typical smut fungi, with three distinct stages (Zhang et al., 2017): the budding growth stage of haploid cells from diploid teliospore germination; the mating stage, which is a prerequisite for infection; and pathogenicity development, with proliferation and teliospore formation happening in planta. The mating stage includes two steps: fusion of two compatible 
haploid cells, mainly regulated by a pheromone receptor system that consists of pheromones and pheromone receptors (encoded at the triallelic a mating-type locus), and post-fusion filamentation that is capable of infection, controlled by the active heterodimeric bE/bW complex (Liang et al., 2019; Zhang et al., 2019). The $\mathrm{T}$ type strain causes gray jiaobai, which is discarded by farmers due to its unacceptable taste and potential to trigger hypersensitivity pneumonitis (Fujii et al., 2007). However, there are also galls with a white appearance, called white jiaobai in China. These are valued by farmers, and contain an inner tissue full of fungal hyphae, probably due to colonization by the MT type strain (Ye et al., 2017). It is widely believed that in white jiaobai, $U$. esculenta only grows within the stems during plant development and overwinters in the rhizomes that are left for reproduction (Zhang et al., 2012; Jose et al., 2016). Studies have found that the MT type strain, which shows an endophytic life in the host, has defects at every stage of its typical life cycle, showing a multi-budding morphology, a slower growth rate in haploid cells, delayed conjugation tube formation and confined hyphal growth during mating, and an attenuated ability to proliferate and form teliospores (Zhang et al., 2017). However, knowledge of the molecular basis for the pathogenic development of $U$. esculenta is very limited.

Pathogenic development in smut fungi is closely related to mating. Its mating system consists of both $a$ and $b$ matingtype loci (Bakkeren et al., 2008; Raudaskoski and Kothe, 2010; Zuo et al., 2019). The recognition of pheromones by opposite pheromone receptors, encoded at the $a$ locus, elicits the fusion of two compatible haploid cells (Szabo et al., 2002; Zhang et al., 2019). The heterodimeric transcription factor bE/bW, which is encoded by the $b$ locus, triggers filamentation and pathogenicity (Yan et al., 2016; Zhang et al., 2019). The cross-talk between the highly conserved cAMP pathway and the mitogenactivated protein kinase (MAPK) pathway is crucial for mating by integrating pheromone signaling and environmental cues (Feldbrügge et al., 2006).

In eukaryotic cells, the MAPK signaling pathways are involved in the regulation of developmental processes through the transduction of extracellular signals (Hamel et al., 2012; Jiang et al., 2018). Five MAPK pathways have been identified in Saccharomyces cerevisiae, among which the Fus3/Kss1 MAPK pathway, an evolutionarily conserved MAPK module, is responsible for mating, filamentous growth, and invasive growth (Zhao et al., 2007). In fungi, Fus3/Kss1 homologs are conserved in an activation loop (the A-loop), including the TXY dual phosphorylation site, which is phosphorylated by upstream MAPKK and is essential for kinase activity (Chen et al., 2001). However, the mechanism of regulation of Fus3/Kss1 homologs is not conserved in fungi, due to the different needs relating to different environments.

In $S$. cerevisiae, Kss1 responds to starvation signaling by regulating filamentous growth through Ste12, which is tethered by Tec1 to TCS elements upstream of filamentation genes (Chou et al., 2006). Fus3, responding to a pheromone, activates Far1, a bifunctional protein required for polarization and $G(1)$ arrest to repress $\mathrm{G}(1)-S$ specific transcription (Breitkreutz et al., 2001). Together with Kss1, it also activates Ste12, a transcription factor that triggers mating processes by regulating mating genes through the Ste12 binding site (Breitkreutz et al., 2001). Additionally, Fus3 can be autophosphorylated by allosteric Ste5, resulting in downregulation of transcriptional output responding to pheromone signaling, ensuring a tuned quantitative pathway through its input-output property (Bhattacharyya et al., 2006).

In Ustilago maydis, a homolog of the yeast Fus3/Kss1 MAPK pathway has been identified, consisting of MAPKKK Kpp4, MAPKK Fuz7, and MAPK Kpp2/Kpp6, responding to pheromone signaling or plant surface signals to regulate the formation of filamentous dikaryons and fungal virulence (Vollmeister et al., 2012). It directly regulates Prf1, both at the transcriptional and the post-transcriptional level, through promoter discrimination phosphorylation; this activates a defined pheromone-responsive linear transcriptional cascade $\mathrm{bE} / \mathrm{bW}>\mathrm{Rbfl}$, which is essential for filamentous growth and further pathogenic development (Kaffarnik et al., 2003; Zarnack et al., 2008; Vollmeister et al., 2012). Rbf1 may also be directly induced by Prf1, which is regulated by activated Kpp2 (Zarnack et al., 2008). However, this does not seem important, and the details of the regulation mechanism are not known. In addition, Kpp2 is involved in the regulation of pheromone-induced cell cycle arrest in the G2 phase and the formation of conjugation tubes, independently of Prf1 (Garcia-Muse et al., 2003).

There are also reports of Fus3/Kss1 homologs in other pathogenic fungi. In Sporisorium scitamineum, SsKpp2 is required for mating and filamentation. This occurs through the integrated regulation of the conserved pheromone signal transduction pathway and fungal quorum-sensing (QS) signal (Deng et al., 2018). In Tilletia indica, TiKpp2 is induced by host factors in a time-dependent manner and participates in myelination growth and pathogenicity by activating the downstream transcription factor Prf1 (Gupta et al., 2013). In Magnaporthe oryzae, Pmk1 is responsible for appressoria formation and cell-to-cell invasion by responding to plant cues (Zhao et al., 2005; Sakulkoo et al., 2018). In Candida albicans, Cek1 and Cek2 are functionally redundant in the dimorphic switch process, virulence, and cell wall integrity (Correia et al., 2016).

In $U$. esculenta, we identified UeFuz7 and UePrf1, which participate in mating and filamentation (Zhang et al., 2018b). In addition, we identified the Fus3/Kss1 homolog UeKpp2, which interacts with UeFuz7 and UePrf1 and is induced by mating and infection (Zhang et al., 2018a). In this study, we explored the functional properties of UeKpp2 in the life cycle of U. esculenta, including budding growth, stages of mating, and the development of pathogenicity.

\section{MATERIALS AND METHODS}

\section{Strains and Plant Growth Conditions}

The Escherichia coli strain JM109 (Takara) was used for cloning purposes. The compatible haploid T type strains UeT14 (a1b1 CCTCC AF 2015016) and UeT55 (a2b2 CCTCC AF 2015015) and their derivatives (listed in Supplementary Table S1) were used in this study. The strains of U. maydis used in this study are 
also listed in Supplementary Table S1. The growth conditions and media for E. coli (Russell and Sambrook, 2001), U. maydis (Holliday, 1974), and U. esculenta (Zhang et al., 2019) have previously been described. The growth conditions of Zea mays (the early golden bantam) and the wild Z. latifolia used for pathogenic development assays of tested strains before and after stem injection have previously been described (Flor-Parra et al., 2007; Zhang et al., 2019).

\section{Plasmid and Strain Construction}

In the deletion of genes in U. esculenta, a PCR-based approach using hygromycin as the resistance marker was used as previously described (Yu et al., 2015). With the UeT14 genomic DNA as template, the $\sim 1 \mathrm{~kb}$ long left-border and right-border fragments adjacent to the target gene were amplified by PCR, using the primer pairs gene-UF/UR and gene-DF/DR. Both primers have $\sim 25$ bp specific homology arms of hygromycin resistance genes. The hygromycin resistance gene with its promoter was separated into two fragments (up and down), with a $\sim 450$ bp overlap, using PCR with the primers HygF/Hyg3 and Hyg4/Hyg-R, respectively. Then, the left (right) border fragments of the target gene were ligated to the $5^{\prime}$ end (the $3^{\prime}$ end) of the up (down) fragment of the hygromycin resistance gene via fusion PCR using the primer pairs geneUF/Hyg3 (Hyg4/gene-DR). The two resulting fragments were transformed into protoplast cells of distinct $U$. esculenta strains to generate target gene deletion strains through homologous recombination, following a $\mathrm{PEG} / \mathrm{CaCl}_{2}$-mediated protoplast transformation method (Yu et al., 2015). First, the primer pairs gene-verity-F/R for object gene detection, Hyg-verity-F/R for hygromycin resistance gene detection, and gene-F3/MF167 and MF168/gene-R3 for insertion site detection were used in the preliminary screening of transformants. qRT-PCR (with the primer pair gene-QF/R) and Southern hybridization (PCR-probe amplified with primers of Hyg-verity-F/R and gene-verity-F/R) were used for further confirmation.

For complementation of the U. maydis strain SG200 $\Delta \mathrm{kpp} 2$, the $K p p 2$ gene promoter sequence was cloned from genomic DNA of the haploid solopathogenic U. maydis strain SG200 with primers PF1/PR1 (or PF1/PR2). Then the cDNA of the $U$. esculenta strain UeT14 (or the U. maydis strain SG200) was used as template to amplify the open reading frame of UeKpp2 (or Kpp2) using primers UeKpp2-CF/CR (or Kpp2CF/CR). The plasmid P123 was linearized by HindIII and NotI to the $4.6 \mathrm{~kb}$ genomic region. The above three fragments were recombined using ClonExpress ${ }^{\circledR}$ II MultiS One Step Cloning Kit (Vazyme, C113-01) and transformed into E. coli to obtain a plasmid, which was linearized by SspI and transformed into the $U$. maydis strain SG200 $\Delta \mathrm{kpp} 2$ to generate the strains SG200 $\Delta$ kpp2:UeKpp2 and SG200 $\Delta$ kpp2:Kpp2. To complement the UeKpp2 deletion strain, UeKpp2 open-reading frame was PCR amplified using the primer pairs UeKpp2-CF1/CR and cloned into plasmid pUMa932 between the NcoI and NotI sites using ClonExpress ${ }^{\circledR}$ II MultiS One Step Cloning Kit (Vazyme, C113-01). Similarly, UeUkc1 open-reading frame was PCR amplified and plasmid pUMa932-UeUkc1 was generated. The resulting two plasmids were linearized by NdeI and transformed into UeT14 $14 \mathrm{kpp} 2$ and UeT55 $\Delta \mathrm{kpp} 2$ to generate the strains UeT14 $\Delta$ UeKpp2:UeKpp2, UeT55 $\Delta$ UeKpp2:UeKpp2, UeT14 $\triangle$ UeKpp2:UeUkc1, and UeT55 $\triangle \mathrm{UeKpp} 2: \mathrm{UeUkc1}$. The transformants were selected using regeneration agar containing carboxin. The selected transformants were further confirmed based on gene expression levels.

To generate the strains UeTSP $\Delta \mathrm{UeKpp} 2: \mathrm{P}_{\mathrm{b}} \mathrm{UeRbf1}$, adopting the genomic DNA of UeT14 as a template, $\sim 1 \mathrm{~kb}$ left border and right border fragments adjacent to UeKpp2 were amplified by PCR using the primer pairs UeKpp2-UF/UR and UeKpp2DF1/DR. These primers have $\sim 20$ bp specific homology arms of the $5^{\prime}$ end of the hygromycin resistance genes and of the $3^{\prime}$ end of the UeRbf1 gene. Additionally, the promoter of the $b W 2$ gene, the open reading frame of the UeRbf1 gene, and the hygromycin resistance genes were PCR amplified using primer pairs bW2$\mathrm{PF} / \mathrm{PR}$, UeRbf1-CF/CR, and Hyg-F/Hyg-R. Then these were cloned and ligated by fusion PCR. Next, the fusion fragment was separated into two fragments (up and down) with a $\sim 450$ bp overlap, using PCR amplification and the primers Hyg-F/Hyg3 and Hyg4/Hyg-R, respectively. Finally, the up fragment was ligated to the left border fragments of $U e K p p 2$, and the down fragment was ligated to the right border fragments of UeKpp2, using fusion PCR with the primer pairs UeKpp2-UF/Hyg3 (Hyg4/UeKpp2-DR). As with the gene-deletion process, the two constructed fragments were transformed into $U$. esculenta protoplast and analyzed via PCR/RT-PCR/Southern blot to confirm the replacement of UeKpp2 by UeRbf1 in the UeTSP strain, its single insertion, and its expression levels.

All primers used above are listed in Supplementary Table S2.

\section{Mating Assays}

The mating assays of $U$. esculenta were performed following Zhang et al. (2017). Haploid isolates were collected by centrifugation after liquid expansion of the culture and adjusted to an $\mathrm{OD}_{600}$ of $\sim 2.0$. Then equal amounts of compatible test strains were mixed. Next, $2 \mu \mathrm{L}$ drops of this mixture were cultured on YEPS solid medium ( $2 \mu \mathrm{L} U$. maydis SG200 and its derivatives were spotted on PDA solid medium) and cultured at $28^{\circ} \mathrm{C}$ for $60 \mathrm{~h}$ observation at $12 \mathrm{~h}$ intervals.

\section{Plant Infection Assays}

For $U$. esculenta inoculation assays, 20-day-old seedlings of $Z$. latifolia were used. Following Zhang et al. (2017), compatible strains with an $\mathrm{OD}_{600}$ of $\sim 2.0$ were mixed at a 1:1 ratio and syringe-inoculated into seedlings, which then were cultured in a greenhouse under a $12 / 12 \mathrm{~h}$ light/dark cycle at $25 \pm 2{ }^{\circ} \mathrm{C}$ and $70 \%$ relative humidity. For the infection of maize seedlings, SG200 and its derivatives were cultured and resuspended in water to an $\mathrm{OD}_{600}$ of $\sim 2.0$; then, samples were syringe-inoculated into 7-day-old maize seedlings, following Gillissen et al. (1992).

\section{Light Microscopy and Confocal Microscopy}

For microscope observation of cell morphology, we used an inverted microscope (Nikon Ti-S inverted microscope, NT-88-V3 micro-operating system). For colony morphology 
observation, we used a stereo microscope (Nikon stereo microscope). A confocal microscope (Leica Microsystems) was used to examine fungal colonization of the leaf sheath. Fungal hyphae were stained with wheat germ agglutinin-Alexa Fluor 488 (WGA, Sigma, L4895). Samples from infected plants were destained with ethanol and placed into $10 \% \mathrm{KOH}$ at $85^{\circ} \mathrm{C}$ for $3 \mathrm{~h}$, washed twice with PBS ( $140 \mathrm{mM} \mathrm{NaCl}, 16 \mathrm{mM} \mathrm{Na}_{2} \mathrm{HPO}_{4}, 2 \mathrm{mM}$ $\mathrm{KH}_{2} \mathrm{PO}_{4}$, and $3.75 \mathrm{mM} \mathrm{KCl}, \mathrm{pH} 7.5$ ), and vacuum-infiltrated with PBS containing $10 \mu \mathrm{g} \mathrm{mL}^{-1} \mathrm{WGA}$ for $20 \mathrm{~min}$ at intervals of $10 \mathrm{~min}$, following Doehlemann et al. (2008). WGA Fluor 488 was excited at $488 \mathrm{~nm}$, and emitted fluorescence was detected in the 495-530 $\mathrm{nm}$ range. The images were processed using LAS-AF software (Leica Microsystems).

\section{Real-Time PCR}

Real-time PCR was conducted to detect gene expression. Samples of distinct strains from the budding growth stage, the mating stage, and the infection stage were collected at the selected relevant times. CFX Connect ${ }^{\mathrm{TM}}$ Real-Time System (Bio-Rad, United States) was used in combination with Platinum SYBR Green qPCR Premix EX TaqTM (TliRNaseH Plus) (Takara, Japan) for detection, and iCycler software (Bio-Rad) was used for data analyses. $\beta$-Actin was used as the internal reference for measuring gene expression. The experiment had three biological and three technical replicates. Relative expression was determined using the $2^{-\Delta \mathrm{Ct}}$ method, with values of $p<0.05$ considered significant. All primers are listed in Supplementary Table S2.

\section{RESULTS}

\section{Functional Complementation Between UeKpp2 of $U$. esculenta and Kpp2 of $U$. maydis}

UeKpp2 in $U$. esculenta has an amino acid identity of $96 \%$ to Kpp2 in $U$. maydis, and the TEY dual phosphorylation sites are conserved (Zhang et al., 2018a). Cross-species complementation assays were carried out to explore the homologies of UeKpp2 to $\mathrm{Kpp} 2$ in terms of function. The coding sequences for UeKpp2 and Kpp2 were introduced into the U. maydis SG200 $\Delta \mathrm{kpp} 2$ strain (Müller et al., 2000), under the native promoter of Kpp2 to exclude problems with promoter strength or time of expression. The derived single-copy strains SG200 $\Delta \mathrm{kpp} 2: \mathrm{Kpp} 2-$ 3 and SG200 $\Delta$ kpp2:UeKpp2-6 were selected after being verified by Southern blot analyses (Supplementary Figure S1). All of the strains, including SG200 and SG200 $\Delta \mathrm{kpp} 2$, were cultured on PDA plates and subjected to a virulence assay. Filamentous growth was inhibited (Figure 1A) and a few tumors were formed (Figure 1B) in SG200 $\Delta \mathrm{kpp} 2$, consistent with previous results that mutation of $K p p 2$ reduces pathogenic development (Müller et al., 2000). However, the SG200 $\Delta \mathrm{kpp} 2: U e K p p 2-6$ and SG200 $\Delta$ kpp2:Kpp2-3 strains appeared as fuzzy colonies in vitro (Figure 1A) and showed severe disease phenotypes after inoculation, comparable to those of SG200 (Figure 1B). These results illustrate that UeKpp2 is capable of complementing the

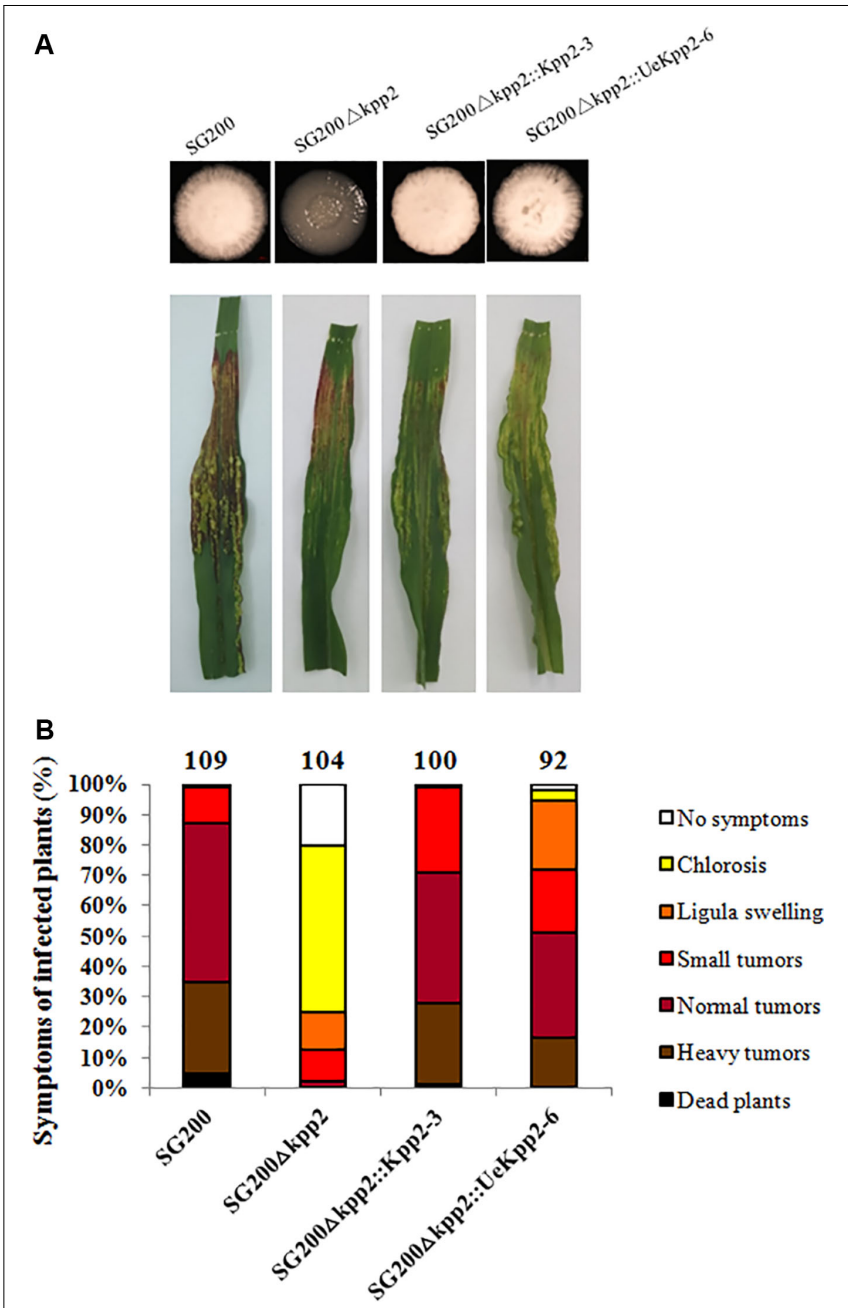

FIGURE 1 | UeKpp2 is capable of complementing the SG200 $\Delta$ kpp2 mutant phenotype. (A) Solopathogenic strains derived from SG200 were tested for filamentous growth on PDA plates. Images were taken after incubation for $72 \mathrm{~h}$ at $28^{\circ} \mathrm{C}$. Filamentous growth appeared white and fuzzy. (B) The disease symptoms and ratings of the solopathogenic strains derived from SG200 are indicated below each column. The strains used to inoculate the 7-day-old corn seedlings are indicated below each column. The disease symptoms were scored 12 days after infection. Photographs of the typical symptoms are given in the top row. The symptoms were grouped into one of seven categories according to the most severe symptoms displayed in a given plant, following Kämper et al. (2006). The groups are color-coded in order of severity on the right of the diagram. The mean values of the three independent infections are indicated above the respective columns, as are the total number of infected plants.

SG200 $\Delta$ kpp2 mutant phenotype, indicating the potential role of UeKpp2 in mating and pathogenicity.

\section{Deletion of UeKpp2 in U. esculenta Alters Budding Cell Morphology}

We separately knocked out UeKpp2 in wild-type strains UeT14 and UeT55, which were selected after validation by Southern blot and qRT-PCR analyses to ensure a single and correct insertion (Supplementary Figure S2). The mutant strains, when grown on 

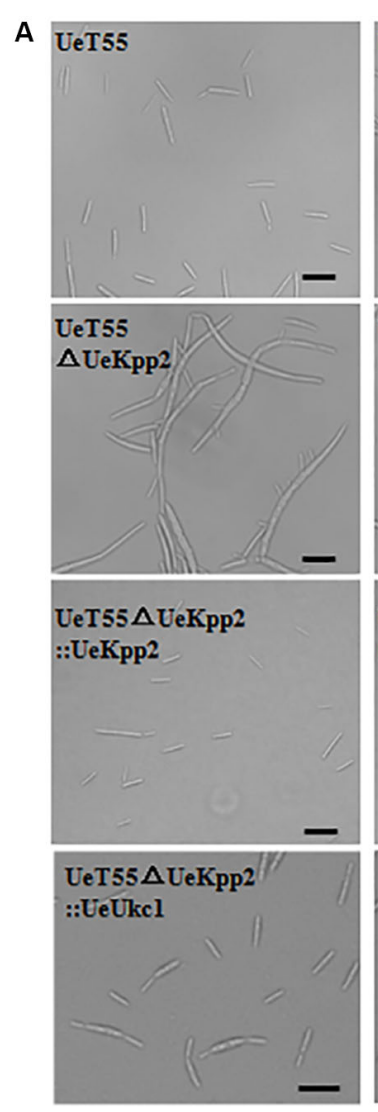
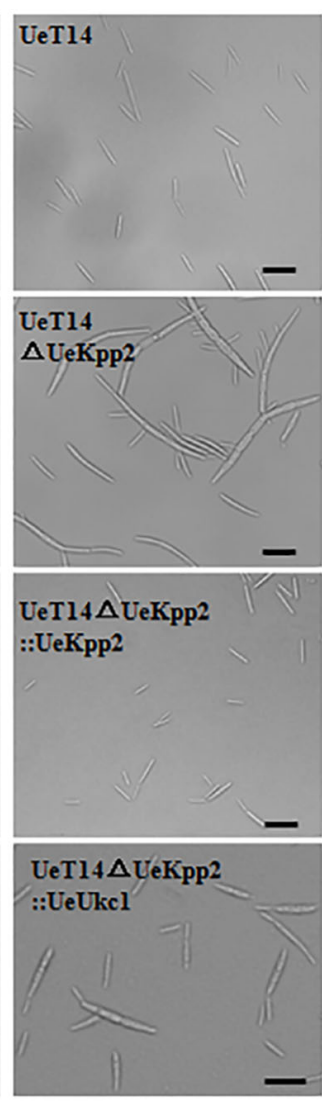

B
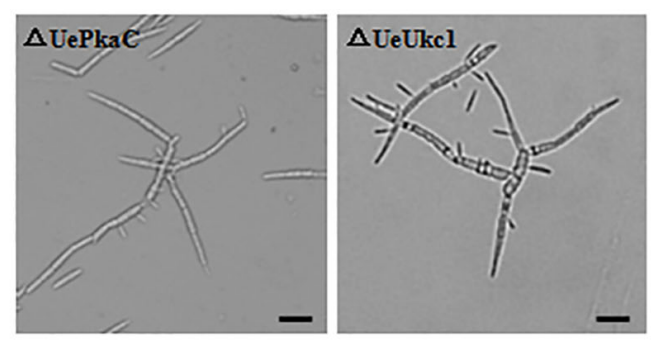

C
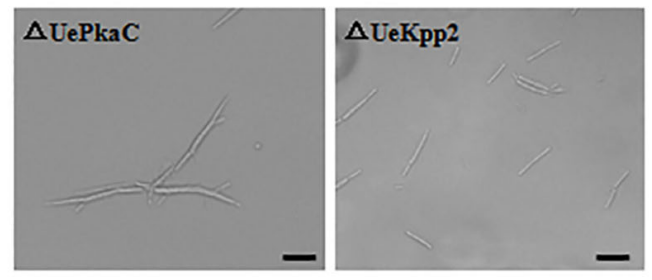

D

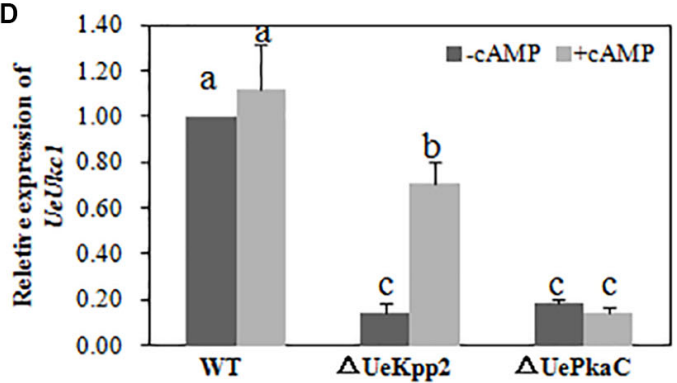

FIGURE 2 | UeKpp2 is involved in the regulation of the morphology of budding cells but not in stress response. (A) Microscopic imaging of budding cells of the WT strains, UeKpp2 mutants, UeKpp2 reconstituted strains or UeUkc1 over-expressed strain in UeKpp2 mutants, $24 \mathrm{~h}$ after spotted culture on YEPS medium. Scale bar $=20 \mu \mathrm{m}$. (B) Microscopic imaging of budding cells of UePkaC mutant or UeUkc1 mutant in UeT14 strain, $24 \mathrm{~h}$ after spotted culture on YEPS medium. The scale bar $=20 \mu \mathrm{m}$. (C) Microscopic imaging of budding cells of UePkaC mutant or UeKpp2 mutant in UeT14 strain, $24 \mathrm{~h}$ after spotted culture on YEPS medium supplemented with 20 mM cAMP. Scale bar $=20 \mu \mathrm{m}$. (D) Relative expression of UeUkc1 in WT, UePkaC mutant or UeKpp2 mutant, $24 \mathrm{~h}$ after spotted culture on YEPS medium (-CAMP) or YEPS medium supplemented with $20 \mathrm{mM}$ cAMP (+CAMP). The letters above the columns indicated significant differences at $p<0.05$ (Tukey). Each strain was cultured in liquid YEPS medium to an $\mathrm{OD}_{600}$ of $\sim 0.8$ and collected by centrifugation to reach the target concentration of $10^{7}$ cells per mL.

a solid YEPS medium, showed no differences in growth rate from WT strains (Supplementary Figure S3). Nitrogen starvation (BM medium) and stressful conditions, including cell wall stress (0.5 mM Congo red), hyperosmotic stress (500 $\mathrm{mM} \mathrm{NaCl}$ ), and oxidative stress ( $1 \mathrm{mM} \mathrm{H}_{2} \mathrm{O}_{2}$ ) (Deng et al., 2018), slowed cell growth in both the WT strains and UeKpp2 mutants. However, these growth rates did not differ between WT and mutant strains (Supplementary Figure S3).

It is worth noting that the cell morphology of UeKpp2 mutants changed. Compared to the short $(19.4 \pm 5.2 \mu \mathrm{m})$ and yeastlike budding cells of WT strains (Figure 2A), mutant cells were longer $(28.9 \pm 9.5 \mu \mathrm{m})$. In addition, most mutants formed long chains, in which cells were attached to each other or appeared elongated with multiple buds (Figure 2A). Moreover, this aberrant morphology was apparent under normal as well as stressed conditions (data not shown). To ensure that all potential mutant phenotypes were associated with this mutation, we also created reconstituted UeKpp2 strains by restoring the ORF of UeKpp2 into the UeKpp2 mutant strain using the constitutive promoter Otef; the results were confirmed using qRT-PCR to ensure transcript restoration (Supplementary Figure S4). Microscopy observation showed that the reconstituted UeKpp2 strains recovered the normal phenotype (Figure 2A), indicating a possible role of UeKpp2 in regulating the shape of yeast-like cells in budding growth.

Further, we found that a mutation of UeUkc1 (accession number: MN845072) or UePkaC (accession number: ALM02104.1), genes we had identified in other studies, caused similar cell shapes to the UeKpp2 mutants (Figure 2B and Supplementary Figure S2). Amino acid sequence analyses showed that UeUkc1 may be homologous to the nuclear Dbf2related (NDR) kinase with a role in determining cell shape (Durrenberger and Kronstad, 1999), and that UePkaC may be homologous to the catalytic subunit of the protein kinase A in the cAMP-PKA signaling pathway (Supplementary Figure S5). Considering that cAMP-PKA signaling is involved in polar growth, which maintains normal cell morphology (Gold et al., 1994; Durrenberger et al., 1998) and cross-talks with MAPK signaling in many cases (Martinez-Espinoza et al., 2004; Meng and Zhang, 2013), we first analyzed the microscopic morphology 
A

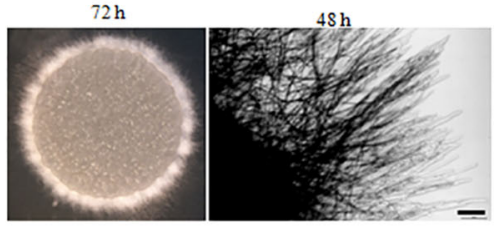

B
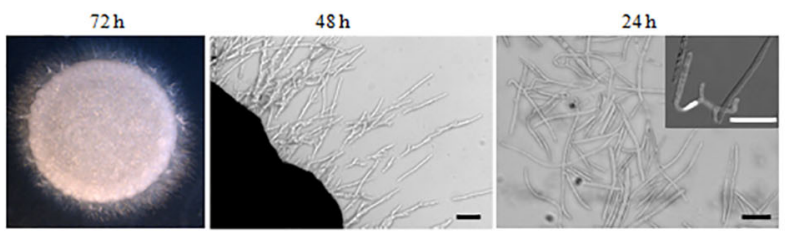

C

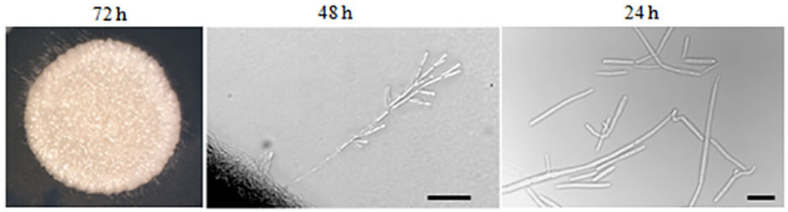

D

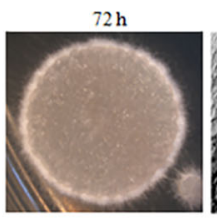

$48 \mathrm{~h}$

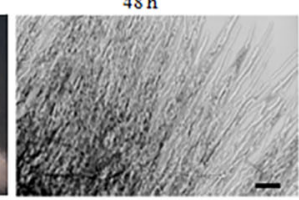

$24 \mathrm{~h}$

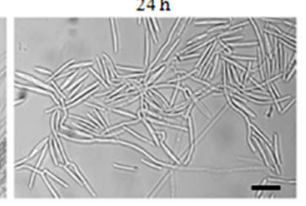

E
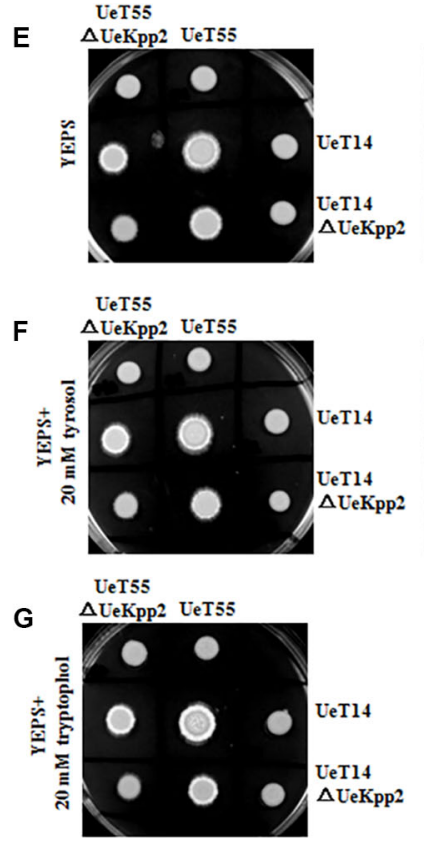

UeT55

H $\triangle$ UeKpp2 UeT55

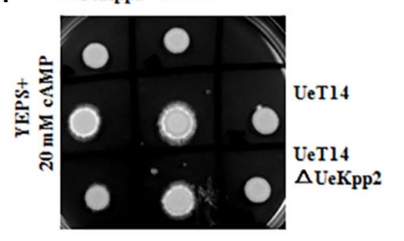

UeT55

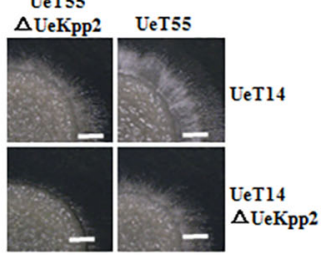

UeT55

$\triangle$ UeKpp2 UeT55

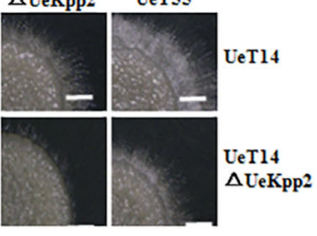

UeT55

$\triangle$ UeKpp2 UeT55

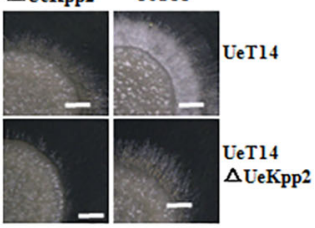

UeT55

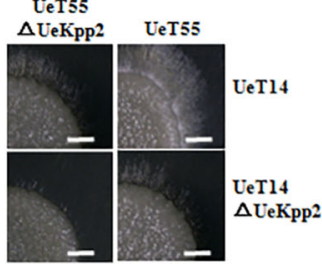

FIGURE 3 | Deletion of UeKpp2 impaired mating and filamentous growth. The morphology of colonies, hyphae, or cell fusion in the compatible WT strains UeT14

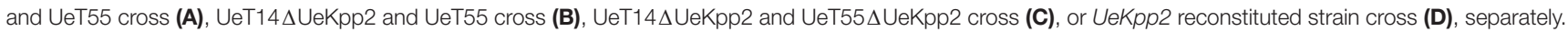
They were spotted onto YEPS medium. Photographs were taken at $72 \mathrm{~h}$ under inverted microscope and at 48 and $24 \mathrm{~h}$ under a stereo microscope. The scale bar represents $100 \mu \mathrm{m}$ in $48 \mathrm{~h}$ and $20 \mu \mathrm{m}$ in $24 \mathrm{~h}$. The impact of QSMs and cAMP on mating and filamentous growth of WT and Uekpp2 mutant was assessed in (E) YEPS solid medium, (F) YEPS supplemented with 20 mM tyrosol, (G) YEPS supplemented with 20 mM tryptophol, or (H) YEPS supplemented with 20 mM CAMP. The images on the left are colonies images in a culture dish taken by a camera at $96 \mathrm{~h}$. The images on the right show the clearer hyphae growth status at $72 \mathrm{~h}$ by stereomicroscopy.

of UeKpp2 under cAMP treatment. UeKpp2 mutants recovered normal cell shape in the YEPS medium when supplied with $20 \mathrm{mM}$ cAMP, while the UePkaC mutants did not (Figure 2C). Also, because downregulation of $u k c 1$ results in a prolonged G2 phase, which leads to a change in cell shape (Sartorel and Perez-Martin, 2012), we assessed UeUkc1 expression in UeKpp2 and $U e P k a C$ mutants cultured for $24 \mathrm{~h}$ in YEPS medium or YEPS medium with $20 \mathrm{mM}$ cAMP. There was an extremely significant reduction of UeUkcl in the mutants compared to WT strains. After cAMP treatment, the expression of $U e U k c 1$ was significantly upregulated in the UeKpp2 mutants while there were no obvious expression changes in UePkaC mutants (Figure 2D). Furthermore, the cell shape of UeKpp2 mutants recovered, whereas that of $U e P k a C$ mutants did not (Figure 2D), indicating that effective expression of $U e U k c 1$ is essential to maintain normal cell morphology. In addition, we improved the expression level of $U e U k c 1$ in the UeKpp2 mutant by over-expressing UeUkc1 under the constitutive promoter Otef (Supplementary Figure S4). The constructed strain showed a normal phenotype (Figure 2A). These findings indicated that UeKpp 2 might regulate transcriptional induction of $U e U k c 1$ in cell shape regulation.

\section{Deletion of UeKpp2 in U. esculenta Impairs Mating and Filamentous Growth in vitro}

The mating and filamentous growth of UeKpp2 mutants were assessed in vitro by co-spotting compatible combinations on YEPS plates. The compatible WT strains UeT14 and UeT55 with opposite mating types served as a positive control, where a white fuzzy appearance at the edge of the colony indicated successful mating and the formation of filaments (Figure 3A). However, filamentous growth was obviously inhibited in the compatible combination of UeKpp2 mutants (Figure 3C), and recovered to a comparable level of control in the reconstituted strains (Figure 3D). The crosses between UeT14 $\Delta$ UeKpp2 and UeT55 or between UeT14 and UeT55 UUeKpp2 showed a delayed 
filamentous growth (Figure 3B). Further follow-up observations showed that normal fusion and filamentous growth appeared $\sim 24 \mathrm{~h}$ after culturing in WT strains (Figure 3A), while rare fusion cells appeared in the double mutant cross (Figure 3C) or in the cross between UeT14 $\Delta$ UeKpp2 and UeT55 (Figure 3B). We also observed unusually long and branched conjugation tubes or branched and curly filaments in the cross of UeT14 $\Delta$ UeKpp 2 and UeT55 (Figure 3B) but relatively little formation of conjugation tubes in the double mutant cross, although cell fusion was not affected (Figure 3C). These findings indicate a defect in conjugation tube formation in UeKpp2 mutants. Moreover, the radial filamentous growth almost disappeared in the double mutant cross and was impaired in the UeT14 $\Delta$ UeKpp2 and UeT55 cross. Instead, hyphal tips were branched (Figures 3B,C). This phenomenon suggests that UeKpp2 also plays a role in filamentous growth.

In addition, we tested the effects of the cAMP-PKA signaling or fungal QSM signaling compounds tryptophol and tyrosol (Chen and Fink, 2006; Wongsuk et al., 2016) on the mating and filamentous growth of UeKpp2 mutants (Figures $3 \mathrm{E}-\mathbf{H}$ ). The addition of tryptophol led to white aerial mycelium growing denser in the two compatible WT strains. However, it did not promote or restore mating or filamentation growth in UeKpp2 mutants, where the defects of conjugation tube formation and radial filamentous growth appeared. On the other hand, cAMP, which restored the morphology of budding cells in UeKpp2 mutant, had no impact on the mating/filamentous growth of this mutant.

\section{Delayed Formation of Conjugation Tubes in UeKpp2 Mutants Is Not Related to a Gene Induction}

Earlier experiments have shown that the defect in the mating of UeKpp 2 mutant is mainly related to the formation of conjugation tubes. Furthermore, we introduced the EGFP over-expression strain UeT55-EGFP to examine the conjugation tubes in the crosses between UeT14 14 UeKpp2 and UeT55-EGFP, under a six-interval microscopic observation. Before $18 \mathrm{~h}$ culture, all of the conjugation tubes that were observed had formed in the green fluorescent cells (Figure 4A). This indicated that the delayed formation of the conjugation tubes only occurs in UeKpp2 mutant cells.

As noted, the pheromone signaling pathway, including the UePrf1 and a genes, is important for regulating conjugation formation (Zhang et al., 2018b). Thus, the expression levels of the UePrf1 and $a$ genes were checked in the double mutant cross compared to the WT strain cross, using qRT-PCR. Mutation of UeKpp2 did not change the basic or induced expression levels of the genes tested at $12 \mathrm{~h}$ (Figures 4B-F). Interestingly, the $m f a$ genes maintained high expression levels after being cultured for $24 \mathrm{~h}$, although the levels decreased in controls (Figures 4CF). Furthermore, the expression of UePrf1 was induced to a higher level in mutants at $24 \mathrm{~h}$ than in WT strains (Figure 4B). These findings indicate that the delayed formation of conjugation tubes in UeKpp2 mutants, which was not related to the defect in induction of $a$ genes, has an unknown cause. It was also worth investigating the relationship between the prolonged induced expression of both UePrf1 and $a$ genes and the malformation of conjugation tubes and hyphae.

\section{Defective Filamentous Growth in UeKpp2 Mutants Is Related to the Defect in UeRbf1 Induction}

Induced expression of either the active bE/bW heterodimer or Rbf1 triggers a dimorphic switch from budding yeast-like growth to filamentous tip growth in the typical smut fungi $U$. maydis (Heimel et al., 2010a,b). In U. esculenta, the UeRbf1 and $b$ genes also participate in filamentous growth ( $\mathrm{Hu}$ et al., 2015; Zhang et al., 2019). First, we detected the expression levels of the UeRbf1 and $b$ genes in the UeKpp2 double mutant cross relative to the WT strain cross during mating, using qRT-PCR. The results showed that the mutation of UeKpp2 did not change the basic or induced expression levels of the genes when the expression levels were compared at 0 and $48 \mathrm{~h}$. Additionally, at $36 \mathrm{~h}$, the expression of $b$ genes was obviously lower in the mutants than in WT strains, indicating delayed induction of $b$ genes in mutants (Figures $\mathbf{5 A - D}$ ). In the fusion process, the delayed induction of $b$ genes may be simply related to delayed cell fusion. Further, the basic expression of $U e R b f 1$ is significantly higher in UeKpp2 mutants than in WT strains, which appeared not to have significantly changed during mating. However, in WT strains during mating, UeRbfl was induced at $24 \mathrm{~h}$, reached its highest level at $36 \mathrm{~h}$, and then fell (Figure 5E). We also studied the UeTSP strain (a2b2: $\left.\mathrm{P}_{\mathrm{bEl}}: \mathrm{bE} 1 \mathrm{P}_{\mathrm{mfa} 1.2}: \mathrm{mfa} 1.2\right)$ (Zhang et al., 2019), which showed filamentous growth and had a fuzzy appearance without mating. Consistent with the expected results, the deletion of UeKpp2 and UeRbf1 in the UeTSP strain showed similarly scarce filament formation (Figure 5F). Interestingly, the expression of $b$ genes in mutants was comparable to that of the WT strains during the culture, but the expression of $U e R b f 1$ was markedly reduced in $U e K p p 2$ mutants, which showed a drastically reduced filamentation (Figure 5G). These results suggest that the mutated UeKpp2 did not impact the expression of the $b$ genes but did influence the induction of UeRbf1. To find support for this conjecture, a $U e R b f 1$ induction strain in the UeKpp2 mutant was constructed under the influence of the promoter of $b W 2$ (UeTSP $\Delta U$ eKpp2:PbUeRbf1). In this strain, UeRbf 1 was induced during the culture (Figure 5G). As expected, it recovered its filamentous growth and fuzzy appearance. All of these results suggest that the defective filamentous growth in $U e K p p 2$ mutants was related to the defect in $U e R b f 1$ induction, not the induction of $b$ genes.

\section{UeKpp2 Is Not Required for Penetration, Proliferation, Teliospore Formation, or Germination in $U$. esculenta}

An inoculation test was carried out to test the pathogenicity of the UeKpp2 mutant. At 3 days post inoculation (dpi), there was infectious hyphal growth of WT strains (mixed with UeT14 and UeT55) but few infectious hyphae in the UeKpp2 deletion mutant. However, at $6 \mathrm{dpi}$, the infectious hyphal growth of WT strain and UeKpp2 mutant were almost identical (Figure 6A). 
A

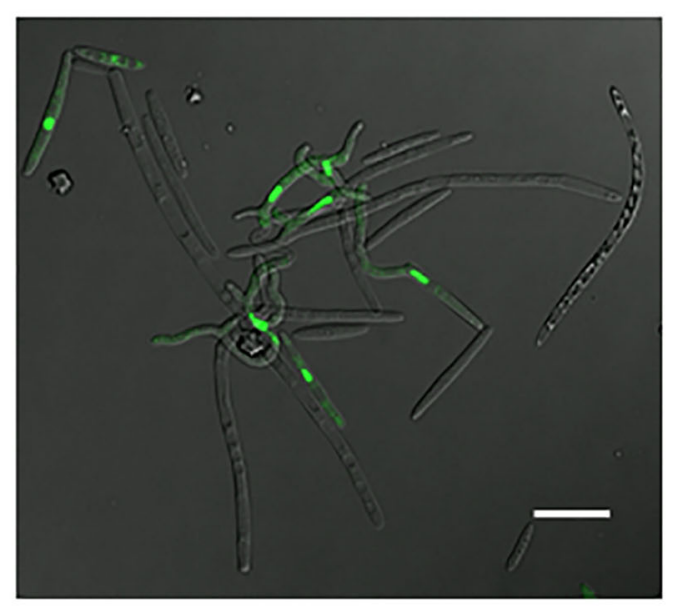

c
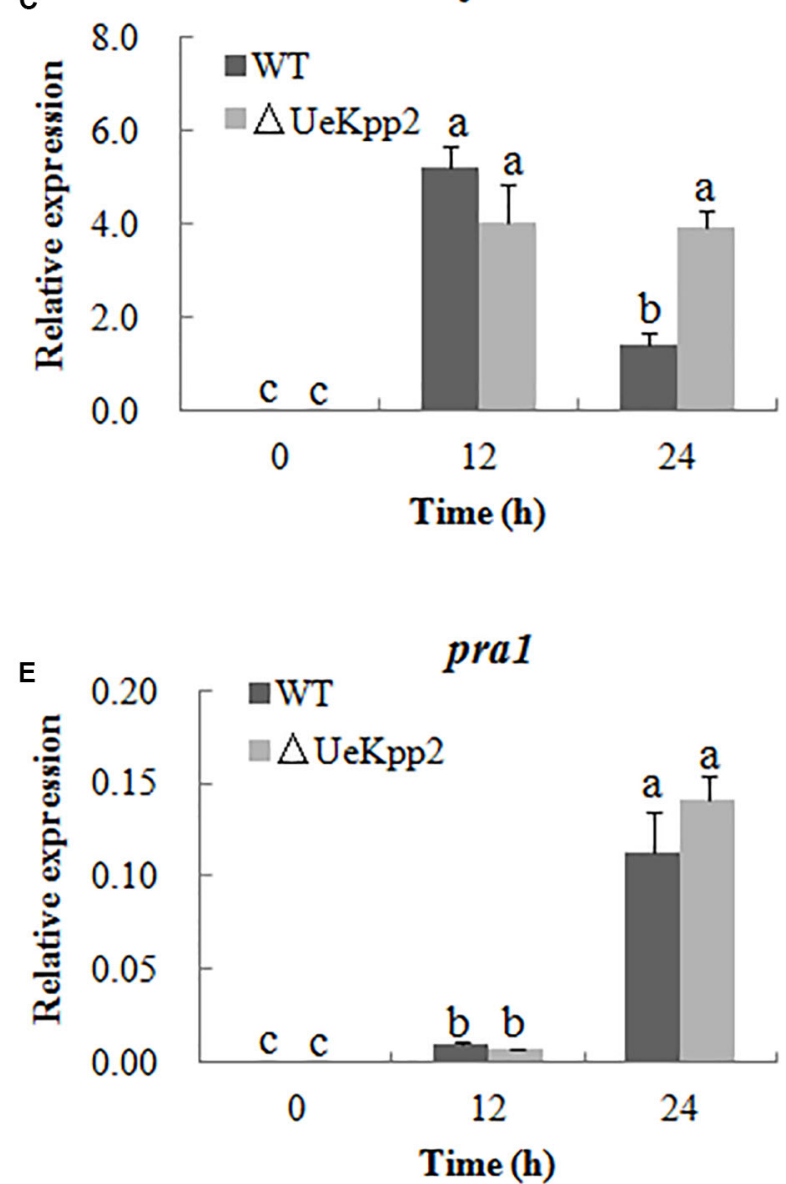

B

\section{UePrf1}

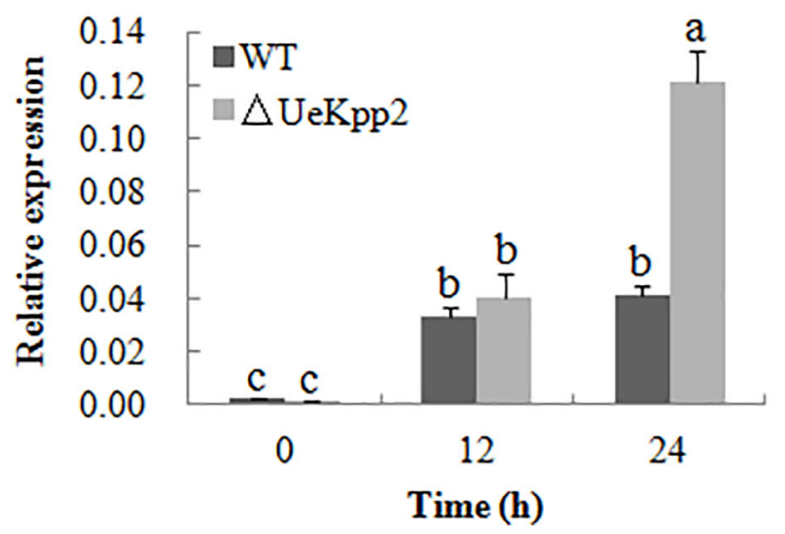

$m f a 2.1$

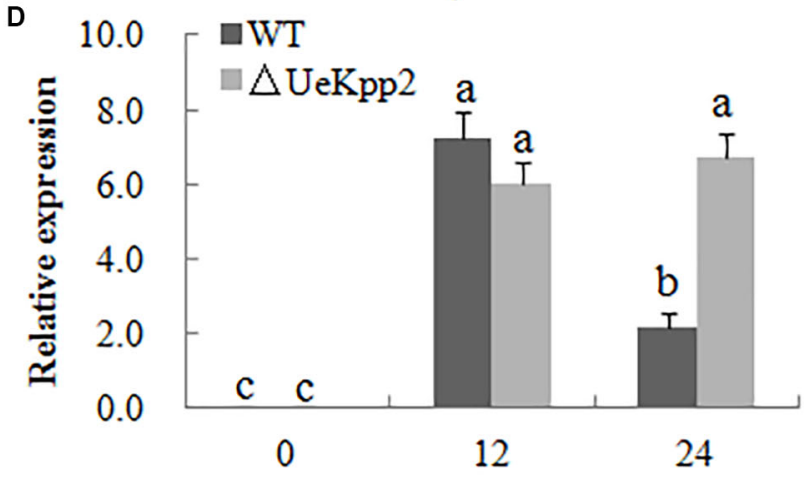

Time (h)

pra2

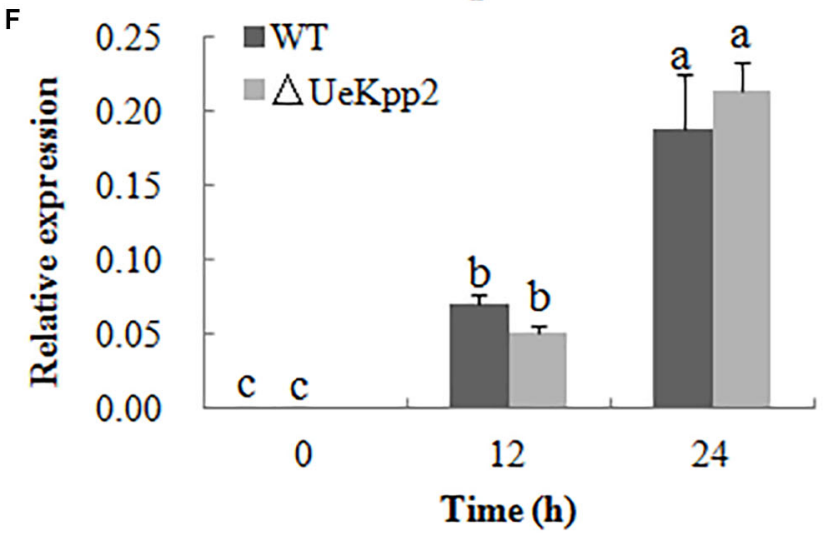

FIGURE 4 | Deletion of UeKpp2 delayed conjugation tubes formation but not impact the a genes and UePrf1 induction. (A) Confocal microscopy of the cells $18 \mathrm{~h}$ after mating of UeT14 $\mathrm{U}$ UeKpp2 and UeT55-EGFP strains cultured on YEPS medium. The conjugation tubes formed in the green fluorescent cells. Scale bar represents $20 \mu \mathrm{m}$. Relative expression of UePrf1 (B), mfa1.2 (C), mfa2.1 (D), pra1 (E), and pra2 (F) in the wild-type strains UeT14 $\times$ UeT55 and the corresponding UeKpp2 deletion strain during mating on YEPS medium. Samples were collected at 0,12 , and $24 \mathrm{~h}$. The letters above the columns indicate significant differences at $p<0.05$ (Tukey). 


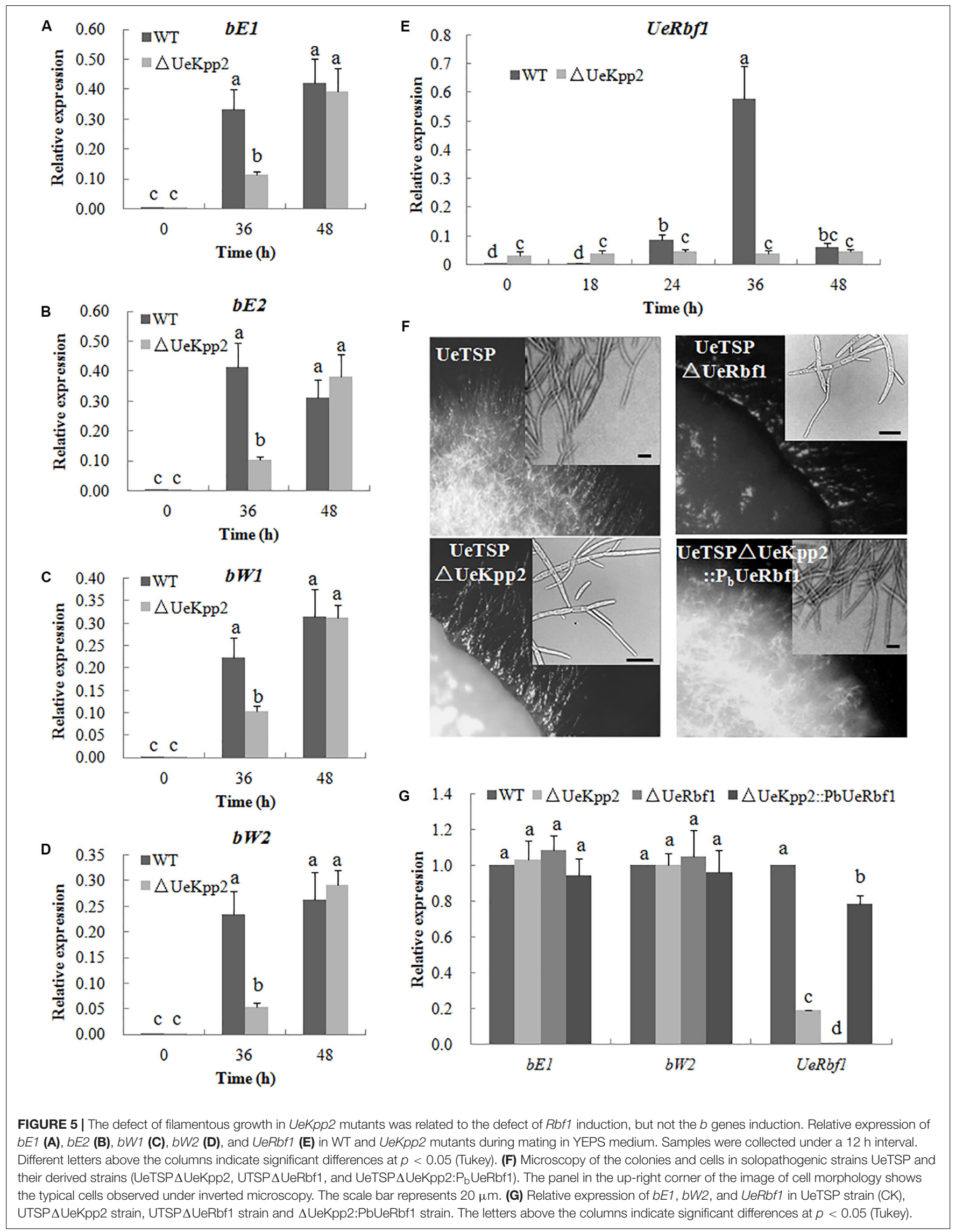


A
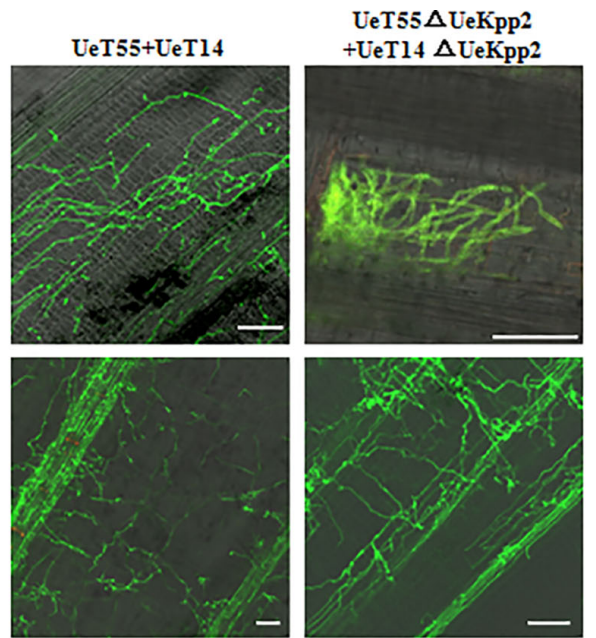

$\mathrm{UeT} 55+\mathrm{UeT} 14$

C

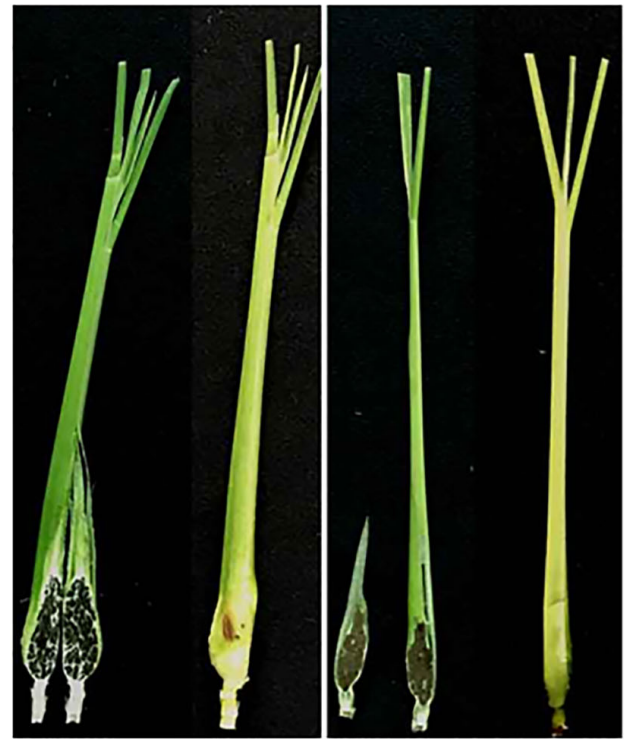

D

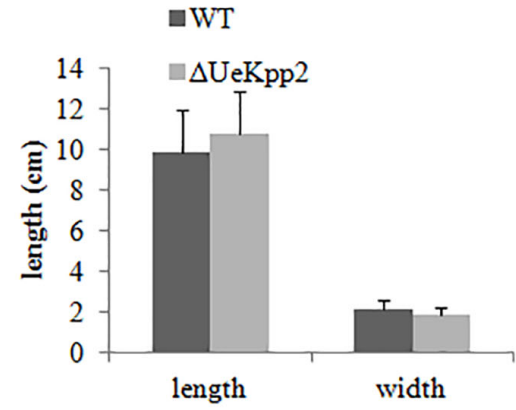

B
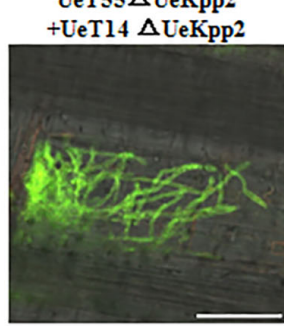

0.20
$0.18-\quad \square \mathrm{WT}$
0.16

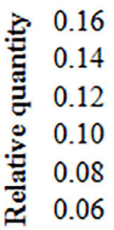

0.04

0.02

0.00

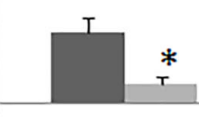

3

Days post inoculation

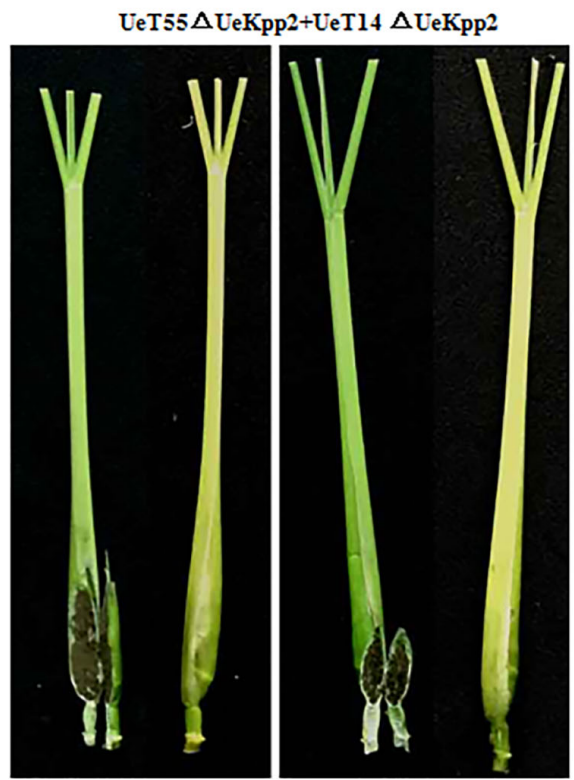

E

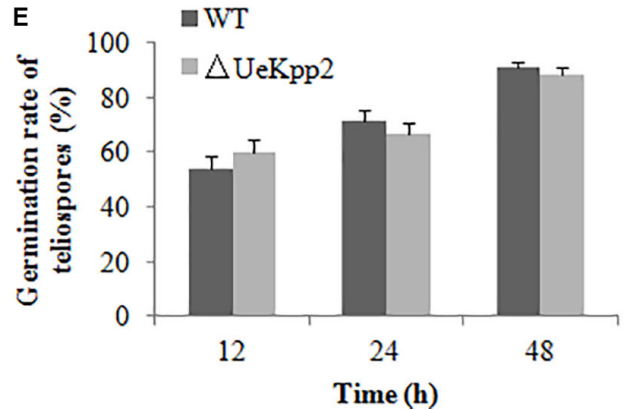

FIGURE 6 | Deletion of UeKpp2 has no influence on penetration, proliferation, teliospore formation, or germination in U. esculenta. (A) Observation of fungal infection status in leaf sheath. Samples infected with the wild-type strains UeT14 $\times$ UeT55 and the corresponding UeKpp2 deletion strains were collected at 3 (above) and 6 dpi (below), stained with wheat germ agglutinin-Alexa Fluor 488 and analyzed using laser scanning confocal microscopy. The scale bar represents $50 \mu \mathrm{m}$. (B) Relative-quantity analyses of the DNA copies of UeActin and ZIActin to evaluate the hyphal amount. The samples were collected at 3 and 6 dpi. Asterisk denotes significant difference at $p<0.05$. (C) Phenotypes of the swollen stems. Samples infected with the wild-type strains UeT14 $\times$ UeT55 (left) and the corresponding UeKpp2 deletion strains (right) were collected at $90 \mathrm{dpi}$. Both formed swollen stems with full of teliospores. (D) The length and width of the swollen stems of the plants infected with the wild-type strains UeT14 $\times$ UeT55 and the corresponding UeKpp2 deletion strains. (E) The germination rate of the teliospores of UeKpp2 mutant and WT strains. An appropriate amount of teliospores were collected from plants after 90 days inoculation by UeKpp2 mutant or WT strains, dispersed in 
FIGURE 6 | Continued

water to get equal concentrations, and incubation on YEPS plates at $28^{\circ} \mathrm{C}$. Light microscopy was taken after 12,24 , and $48 \mathrm{~h}$ culture. The numbers of germination teliospores were analyzed. There were no significant differences in the teliospores germination rate between UeKpp2 mutant and WT strains.

Relative-quantity analyses of the DNA copies of UeActin and ZlActin were used to detect the number of fungal cells to evaluate the amount of hyphae. Fewer cells were detected in the UeKpp2 mutant at $3 \mathrm{dpi}$ (Figure 6B). However, at $6 \mathrm{dpi}$, comparable amounts were observed between the mutant and WT strains (Figures 6A,B). After that, there was no obvious difference of hyphal growth status and amounts observed in the WT strain and UeKpp2 mutant infected plants (data was not shown). Thus, even on the plant surface, the UeKpp2 deletion only influenced infectious hyphal formation and did not impact fungal penetration or proliferation. Moreover, at $75 \mathrm{dpi}$, we observed slightly swollen stems of more than $70 \%$ both of the WT strain infected plants (31/40, swollen plants/total plants) and UeKpp2 mutant infected plants (29/40), but no teliospores were observed in a five randomly selected plants whether infected with WT strain or UeKpp2 mutant. At 80 dpi, we could observe teliospores in all the five randomly selected plants, no clear distinction between the plants infected with WT strain or UeKpp2 mutant. After 90 days of culture, all of the infected plants inoculated with either the UeKpp2 deletion mutant or the WT strains had swollen stems full of teliospores, with similar size and shape (Figures 6C,D). Additionally, the germination rate of the teliospores showed no difference between the mutant and WT strains (Figure 6E). These results indicate that UeKpp2 was not required for penetration, proliferation, teliospore formation, or germination in U. esculenta.

\section{DISCUSSION}

In smut fungi, the switch from yeast-like budding growth to infective filamentous growth occurs in response to environmental cues and is tightly controlled by complex genetic pathways. Among these, the cAMP-PKA and MAPK pathways are crucial to ensuring the coordination and timing of the processes (Skalhegg and Tasken, 2000; Breitkreutz et al., 2001; Martinez-Espinoza et al., 2004; Raudaskoski and Kothe, 2010). In this work, we studied the functional properties of the MAPK UeKpp2. It should be noted that the ectopic expression of UeKpp2, a highly conserved MAPK in smut fungi, restored defective filamentous growth and pathogenic fungal development of the U. maydis kpp2 mutant SG200 $\Delta \mathrm{kpp} 2$. However, in U. esculenta, UeKpp2 only participates in mating and filamentous growth in vitro and does not impact fungal pathogenicity. In addition, we found that a morphological change appeared in budding cells in the UeKpp2 deletion mutant, indicating a special role for UeKpp2 in the budding growth of U. esculenta.

\section{Regulation of Mating-Type Genes Through MAPK Is Weakened in U. esculenta}

The MAPK and CAMP-PKA signaling pathways are necessary for pheromone responses in fungi (Maidan et al., 2005; Klosterman et al., 2007; Saito, 2010; Jung et al., 2011). In U. maydis, which is closely related to U. esculenta (Ye et al., 2017), Prf1 is a core transcription factor that regulates the expression of the $a$ and $b$ genes during mating (Zhang et al., 2018b). Recognition between pheromones and pheromone receptors results in the activation of MAPK cascade and PKA signaling, in which the MAPK Kpp2 further regulates Prf1 at the transcriptional level through phosphorylation, and PKA catalytic subunits are responsible for the post-transcriptional regulation of Prf1 (Kaffarnik et al., 2003). Among these, Kpp 2 is not necessary for the induction of $a$ genes but acts on the increased expression of the $a$ genes. Additionally, Kpp2 is important for pheromone-induced expression of the $b$ heterodimer (Kaffarnik et al., 2003; Muller et al., 2003; Zarnack et al., 2008; Elias-Villalobos et al., 2011). In U. esculenta, we have shown that UePrf1, a homolog of Prf1, is necessary for the regulation of the $a$ and $b$ genes (Zhang et al., 2018b), indicating a pheromone response mechanism that is similar to that of $U$. maydis. However, the expression level of UePrf1 did not decrease during mating in UeKpp2 mutants (Figure 4B). Moreover, the induction of the $a$ and $b$ genes also did not weaken (Figures 4, 5). In addition, pheromones encoded by the $m f a$ genes in one haploid strain is sufficient for the conjugation tubes formation in its compatible strains (Zhang et al., 2019). So mating between UeT14 $\Delta$ UeKpp2 and UeT55-EGFP induced normally functioning conjugation tubes in the UeT55-EGFP strain (Figure 4A), indicating a normal induction of $m f a$ genes in UeKpp2 mutants. Additionally, the $U e R b f 1$ induction strain in the UeKpp2 mutant under the promoter of $b W 2$ (UeTSP $\triangle U$ UKpp2:PbUeRbf1) recovered the induced expression of $U e R b f 1$, the filamentous growth, and the fuzzy appearance (Figure 5G), indicating that the induction of $b$ genes was not affected in UeKpp2 mutants. These findings suggest that the impact of UeKpp2 on the regulation of matingtype genes was significantly weakened in U. esculenta compared to $U$. maydis. We believe that this is due to an endogenous trend in $U$. esculenta. Because most $U$. esculenta overwinter in mycelium form and reinfect directly with mycelium the next year under asexual cultivation, there is a significantly lower chance that a pheromone response will occur during the life cycle of $U$. esculenta. However, it should be further studied whether this is due to the endogenous life cycle of $U$. esculenta in Z. latifolia or there are other regulatory factors that have not yet been found.

\section{UeKpp2 Is Involved in the Regulation of the Morphogenesis-Related NDR Kinase Pathway in U. esculenta}

In fungi, morphology and the cell cycle are intricately connected (Sartorel and Perez-Martin, 2012). Fungi impose delays or arrests at specific cell cycle stages to enable the cell to adapt to unfavorable stress conditions or to synchronize cell cycle 
progression before mating, which is negatively regulated by MAPK cascades (Carbó and Pérez-Martín, 2010). In response to pheromone recognition, cell cycle arrest regulated by MAPK signaling occurs in the process of budding, leading to the formation of conjugation tubes before cell fusion (Garcia-Muse et al., 2003). In yeasts, for example, cell fusion requires a previous G1 cell cycle arrest, regulated by a Fus3 MAPK cascade via phosphorylation of a cyclin-dependent kinase inhibitor Far1 (Davey, 1998). In U. maydis, the activation of Kpp2 results in a prolonged G2 phase, which is believed to result in polar extension of the cell and the formation of conjugation tubes (Garcia-Muse et al., 2003). As with U. maydis, a mutation in the pheromone response MAPK UeKpp2 significantly reduces the formation of conjugation tubes (Figures 3C, 4A).

Meanwhile, we found that the morphology of the budding cells also changed in the UeKpp2 mutants (Figure 2A), and they became elongated and showed several buddings. This was similar to the effect of a defect in the morphogenesis-related NDR kinase (MOR) pathway that is conserved among different fungi (Maerz and Seiler, 2010). In addition, we found that a mutation of $U e U k c 1$, a gene encoding the homolog to the protein kinase critical in the MOR pathway (Verde et al., 1998; Durrenberger and Kronstad, 1999), caused similar budding cell morphology to the UeKpp2 mutants (Figure 2B). In U. maydis, bud formation takes place during the G2 phase and relies almost entirely on polar growth (Steinberg et al., 2001). Downregulation of $u k c 1$ results in a prolonged G2 phase and enlarged cells that are strikingly polarized (Sartorel and Perez-Martin, 2012). Thus, we further investigated the expression levels of UeUkc1 in UeKpp2 mutants. As expected, the expression of $U e U k c 1$ in the mutant fell dramatically (Figure 2D). It is worth noting that the abnormal morphology of the UeKpp2 mutants could be restored to normal, with an increased expression of $U e U k c 1$, by adding $20 \mathrm{mM}$ cAMP or by inducing $U e U k c 1$ over-expression (Figures $2 \mathbf{A}, \mathbf{C}, \mathbf{D}$ ). These results indicate that, in U. esculenta, UeKpp 2 might be involved in the regulation of the MOR pathway through UeUkc1, unlike in the case of $U$. maydis, in which only crk1 has been proven to be responsible for the morphology of MOR mutants (Sartorel and Perez-Martin, 2012).

In addition, we found that mutant UePkaC cells were elongated and had several buds, with a reduced expression of UeUkcl (Figures 2B,D). cAMP, a signal molecule that activates the PKA pathway (Cherkasova et al., 2003), increased the expression level of $U e U k c 1$ and restored the cell shape to its normal form in UeKpp2 mutants. Thus, we speculated that activating the PKA pathway could compensate for the defective cell shape when the MAPK pathway is disabled. In addition, the abnormal morphology of UeKpp2 mutants was not restored under conditions of nitrogen starvation or stress (including cell wall stress, hyperosmotic stress, and oxidative stress). However, an almost normal budding of the two comparable UeKpp2 mutants was observed during mating (Figure 3C). These phenomena led us to suspect that pheromone signaling may activate the PKA pathway to compensate for the defective cell shape when the MAPK pathway is disabled, where environmental cues do not. However, all of these speculations require further evidence.

\section{Role of UeKpp2 in Induction of UeRbf1 in Filamentous Growth Regulation Is Only Observed in vitro}

In $U$. maydis, $b$-dependently induced $R b f 1$ is required for $b$-dependent filament formation and sufficient for filament formation in the absence of an active bE/bW heterodimer (Heimel et al., 2010a). Further, Rbf1 may be regulated by Prf1 through an unknown mechanism (Heimel et al., 2010a). In $U$. esculenta, the $b$ genes also play an important role in the growth of the dikaryotic filament during mating in vitro and in vivo (Zhang et al., 2019). However, in the UeKpp2 mutant with the UeTSP strain background, which can spontaneously form filaments in vitro and in vivo (Zhang et al., 2019) and avoid the influence of conjugation tube formation, filamentous growth was also seriously affected (Figure 5F). During these developmental steps, the expression of the $b$ genes in the mutants was similar to that in the WT strains, while UeRbf1 expression decreased significantly in mutants (Figures $5 \mathbf{E}, \mathbf{G}$ ). It was believed that the defective filamentous growth in UeKpp2 mutants was related to a defect in the induction of $U e R b f 1$, not in the induction of $b$ genes. As with U. maydis (Heimel et al., 2010a), the deletion of UeRbf1 in UeTSP strains could not form infectious hyphae (Supplementary Figure S6). However, this was different from $U$. maydis in that UeKpp2 deletion mutants with the UeTSP strain did not affect the growth of infectious hyphae in the host at all, and the mutation of UeKpp2 did not affect the induction of galls or the formation or germination rate of teliospores (Figure 6 and Supplementary Figure S6). Hence, in U. esculenta, the role of UeKpp2 in the induction of UeRbf1 independently from $\mathrm{bE} / \mathrm{bW}$ heterodimer only happened in vitro during mating in our study. This may be related to the endogenous life cycle of $U$. esculenta in Z. latifolia, such that the mating process with comparable haploid strains rarely appeared. However, further discussion of how UeKpp2 participates in the distinct developmental stages of the life cycle of U. esculenta in response to different external signals is necessary.

\section{DATA AVAILABILITY STATEMENT}

All datasets generated for this study are included in the article/Supplementary Material.

\section{AUTHOR CONTRIBUTIONS}

$\mathrm{YZ}$ and $\mathrm{ZY}$ conceived and designed the experiments, and wrote and revised the manuscript. YH, QC, YY, and YZ performed the experiments. YH, QC, WX, HC, and XY analyzed the data. YZ and $\mathrm{YH}$ prepared the figures and tables. All authors have read and approved the final manuscript.

\section{FUNDING}

This work was supported by the Natural Science Foundation of Zhejiang Province (LY19C140001) and the National Natural Science Foundation of China (31770828 and 31600634). 


\section{ACKNOWLEDGMENTS}

We thank Prof. Regine Kahmann (Department of Organismic Interactions, Max Planck Institute for Terrestrial Microbiology, Germany) for sharing experimental materials and techniques.

\section{SUPPLEMENTARY MATERIAL}

The Supplementary Material for this article can be found online at: https://www.frontiersin.org/articles/10.3389/fmicb. 2020.01053/full\#supplementary-material

FIGURE S1 | Identification of the derived strains SG200 $\mathrm{kpp} 2::$ Kpp2-3 and SG200 $\Delta$ kpp2::UeKpp2-6. (A) The single-copy case was verified by Southern blot analyses. "M" stands for Hindlll marker. "1" strands for the test strain SG200 $\Delta$ kpp2::Kpp2-3. "2" strands for the test strain SG200 $\Delta$ kpp2::UeKpp2-6. (B) The expression level was analyzed by qRT-PCR. ${ }^{* *}$ denotes significant difference at $p<0.01$.

FIGURE S2 | Identification of the derived mutants. (A-C) Southern blot analyses. "M" stands for Hindlll marker. (A) "1" strands for the test strain UeT14 UUeKpp2. "2" strands for the test strain UeT55 $\Delta$ UeKpp2. "3" strands for the test strain UeTSP $\Delta$ UeKpp2. (B) "2" strands for the test strain UeT55 $\Delta$ UePkaC. "3" strands for the test strain UeT14 $\Delta$ UeUkc1. "4" strands for the test strain UeT55 UUeUkc1. "5" strands for the test strain UeT14 $\Delta$ UePkaC. (C) "1" strands for the test strain UeTSP $\Delta$ UeKpp2::PbUeRbf1. "2" strands for the test strain UeTSP $\Delta$ UeRbf1. (D-G) PCR verification of UePrf1 deletion mutants. "M" stands for DL2000 marker. The fragments that are verified from top to bottom are target gene, hygromycin resistance gene, upstream segment of target gene and downstream segment of target gene. (D) "1" strands for the test strain UeT14. "2" strands for the test strain UeT14 U UeKpp2. "3" strands for the test strain UeT55 $\Delta$ UeKpp2. "4" strands for the test strain UeTSP $\Delta$ UeKpp2. "5" strands for the test strain UeT55. (E) "1" strands for the test strain UeT14 $\Delta$ UePkaC. "2" strands for the test strain UeT55?UePkaC. " 3 " strands for the test strain UeT14. "4" strands for the test

\section{REFERENCES}

Bakkeren, G., Kaemper, J., and Schirawski, J. (2008). Sex in smut fungi: structure, function and evolution of mating-type complexes. Fungal Genet. Biol. 45, S15-S21. doi: 10.1016/j.fgb.2008.04.005

Bhattacharyya, R. P., Remenyi, A., Good, M. C., Bashor, C. J., Falick, A. M., and Lim, W. A. (2006). The Ste5 scaffold allosterically modulates signaling output of the yeast mating pathway. Science 311, 822-826. doi: 10.1126/science.112 0941

Breitkreutz, A., Boucher, L., and Tyers, M. (2001). MAPK specificity in the yeast pheromone response independent of transcriptional activation. Curr. Biol. 11, 1266-1271. doi: 10.1016/s0960-9822(01)00370-0

Carbó, N., and Pérez-Martín, J. (2010). Activation of the cell wall integrity pathway promotes escape from G2 in the fungus Ustilago maydis. PLos Genet. 6:e1001009. doi: 10.1371/journal.pgen.1001009

Chen, H., and Fink, G. R. (2006). Feedback control of morphogenesis in fungi by aromatic alcohols. Genes Dev. 20, 1150-1161. doi: 10.1101/gad.1411806

Chen, Z., Gibson, T. B., Robinson, F., Silvestro, L., Pearson, G., Xu, B., et al. (2001). MAP kinases. Chem. Rev. 101, 2449-2476. doi: 10.1021/cr000241p

Cherkasova, V. A., McCully, R., Wang, Y., Hinnebusch, A., and Elion, E. A. (2003). A novel functional link between map kinase cascades and the Ras/cAMP pathway that regulates survival. Curr. Biol. 13, 1220-1226. doi: 10.1016/s09609822(03)00490-1

Chou, S., Lane, S., and Liu, H. P. (2006). Regulation of mating and filamentation genes by two distinct Ste12 complexes in Saccharomyces cerevisiae. Mol. Cell. Biol. 26, 4794-4805. doi: 10.1128/mcb.02053-05

Chung, K. R., and Tzeng, D. D. (2004). Nutritional requirements of the edible gallproducing fungus Ustilago esculenta. J. Biol. Sci. 4, 246-252. doi: 10.3923/jbs. 2004.246.252 strain UeT55. (F) "1" strands for the test strain UeT14 UUeUkc1. "2" strands for the test strain UeT55 $\Delta$ UeUkc1. "3" strands for the test strain UeT14. "4" strands for the test strain UeT55. (G) "1" strands for the test strain UeTSP. "2" strands for the test strain UeTSP $\Delta$ UeRbf1. "3" strands for the test strain

UeTSP $\Delta$ UeKpp2::PbUeRbf1. (H-K) The expression level of target genes was analyzed by qRT-PCR. ${ }^{* \star}$ denotes significant difference at $p<0.01$.

FIGURE S3 | Assessment of tolerance of WT or UeKpp2 mutant toward stressful conditions. (A) Serially diluted cells (from about $10^{7}$ to $10^{4} / \mathrm{mL}$ ) of WT or UeKpp2 mutant were spotted onto YEPS medium, YEPS supplemented with $\mathrm{H}_{2} \mathrm{O}_{2}$ (1 $\mathrm{mM})$, Congo Red $(0.5 \mathrm{mM})$, or $\mathrm{NaCl}(500 \mathrm{mM})$ and $\mathrm{BM}$ medium separately. Images were separately taken 3 days post spotting.

FIGURE S4 | qPCR verification of the expression level of UeKpp2 (A) or UeUkc1 (B) expressed through the constitutive promoter in UeKpp2 mutants. *Denotes significant difference at $p<0.05$

FIGURE S5 | Amino acid sequences arrangement. (A) Amino acid sequences in the arrangement of UeUkc1 protein (MN845072) and its fungal orthologs: $U$. maydis Ukc1 (AAC09291.1), U. hordei Ukc1(CCF50109.1), Neurospora crassa Cot1 (XP_962150.2), Schizosaccharamyces Pombe Orb6 (NP_593165.1). The black and gray shadows denote identical and conserved residues, respectively. The underline represents STKC-NDR-like-fungal domains. (B) Arrangement of amino acid sequences of UePkaC protein (ALM02104.1) and its fungal orthologs: U. maydis PkaC (XP_011391132.1), Neurospora crassa PkaC (ACA48490.1), Colletotrichum trifolii PkaC (AAC04355.1). The black and gray shadows denote identical and conserved residues, respectively. Underlining represents STKc-PKA-like domains.

FIGURE S6 | Fungal infection status in leaf sheath. Seedlings infected with the UeTSP strain, the corresponding UeKpp2 deletion strain, and the UeRbf1 deletion strain were collected at 3 and $6 \mathrm{dpi}$, stained with wheat germ agglutinin-Alexa Fluor 488 and analyzed by laser scanning confocal microscopy. The scale bar represents $50 \mu \mathrm{m}$.

TABLE S1 | Strains used in this study.

TABLE S2 | Primers used in this study.

Correia, I., Roman, E., Prieto, D., Eisman, B., and Pla, J. (2016). Complementary roles of the Cek1 and Cek2 MAP kinases in Candida albicans cell-wall biogenesis. Future Microbiol. 11, 51-67. doi: 10.2217/fmb.15.127

Davey, J. (1998). Fusion of a fission yeast. Yeast 14, 1529-1566.

Deng, Y. Z., Zhang, B., Chang, C. Q., Wang, Y. X., Lu, S., Sung, S. Q., et al. (2018). The MAP Kinase SsKpp2 is required for mating/filamentation in Sporisorium scitamineum. Front. Microbiol. 9:2555. doi: 10.3389/fmicb.2018.02555

Doehlemann, G., Wahl, R., Horst, R. J., Voll, L. M., Usadel, B., Poree, F., et al. (2008). Reprogramming a maize plant: transcriptional and metabolic changes induced by the fungal biotroph Ustilago maydis. Plant J. 56, 181-195. doi: 10.1111/j.1365-313X.2008.03590.x

Durrenberger, F., and Kronstad, J. (1999). The Ukc1 gene encodes a protein kinase involved in morphogenesis, pathogenicity and pigment formation in Ustilago maydis. Mol. Gen. Genet. 261, 281-289. doi: 10.1007/s004380050968

Durrenberger, F., Wong, K., and Kronstad, J. W. (1998). Identification of a cAMP-dependent protein kinase catalytic subunit required for virulence and morphogenesis in Ustilago maydis. Proc. Natl. Acad. Sci. U.S.A. 95, 5684-5689. doi: $10.1073 /$ pnas.95.10.5684

Elias-Villalobos, A., Fernandez-Alvarez, A., and Ibeas, J. I. (2011). The general transcriptional repressor Tup1 is required for dimorphism and virulence in a fungal plant pathogen. PLos Pathog. 7:e1002235. doi: 10.1371/journal.ppat. 1002235

Feldbrügge, M., Bölker, M., Steinberg, G., Kämper, J., and Kahmann, R. (2006). "Regulatory and structural networks orchestrating mating, dimorphism, cell shape, and pathogenesis in Ustilago maydis," in The Mycota, eds I. Kües and U. R. Fischer (Berlin: Springer-Verlag), 375-391.

Flor-Parra, I., Castillo-Lluva, S., and Perez-Martin, J. (2007). Polar growth in the infectious hyphae of the phytopathogen Ustilago maydis depends on a virulence-specific cyclin. Plant Cell 19, 3280-3296. doi: 10.1105/tpc.107.052738 
Fujii, Y., Usui, Y., Konno, K., Atarashi, K., and Yoshizawa, Y. (2007). A case of hypersensitivity pneumonitis caused by smut spores of Ustilago esculenta. Nihon Kokyuki Gakkai Zasshi 45, 344-348.

Garcia-Muse, T., Steinberg, G., and Perez-Martin, J. (2003). Pheromone-induced G 2 arrest in the phytopathogenic fungus Ustilago maydis. Eukaryotic Cell 2, 494-500. doi: 10.1128/EC.2.3.494-500.2003

Gillissen, B., Bergemann, J., Sandmann, C., Schroeer, B., Bolker, M., and Kahmann, R. (1992). A two-component regulatory system for self/non-self recognition in Ustilago maydis. Cell 68, 647-657. doi: 10.1016/0092-8674(92)90141-x

Gold, S., Duncan, G., Barrett, K., and Kronstad, J. (1994). cAMP regulates morphogenesis in the fungal pathogen Ustilago maydis. Genes Dev. 8, 28052816. doi: $10.1101 /$ gad.8.23.2805

Guo, L. B., Qiu, J., Han, Z. J., Ye, Z. H., Chen, C., Liu, C. J., et al. (2015). A host plant genome (Zizania latifolia) after a century-long endophyte infection. Plant J. 83, 600-609. doi: 10.1111/tpj.12912

Gupta, A. K., Joshi, G. K., Seneviratne, J. M., Pandey, D., and Kumar, A. (2013). Cloning, in silicocharacterization and induction of TiKpp2 MAP kinase inTilletia indicaunder the influence of host factor(s) from wheat spikes. Mol. Biol. Rep. 40, 4967-4978. doi: 10.1007/s11033-013-2597-0

Hamel, L. P., Nicole, M. C., Duplessis, S., and Ellis, B. E. (2012). Mitogen-activated protein kinase signaling in plant-interacting fungi: distinct messages from conserved messengers. Plant Cell 24, 1327-1351. doi: 10.1105/tpc.112.096156

Heimel, K., Scherer, M., Schuler, D., and Kaemper, J. (2010a). The Ustilago maydis Clp1 protein orchestrates pheromone and b-Dependent signaling pathways to coordinate the cell cycle and pathogenic development. Plant Cell 22, 2908-2922. doi: $10.1105 /$ tpc. 110.076265

Heimel, K., Scherer, M., Vranes, M., Wahl, R., Pothiratana, C., Schuler, D., et al. (2010b). The transcription factor Rbf1 Is the master regulator for b-mating type controlled pathogenic development in Ustilago maydis. PLos Pathog. 6:e1001035. doi: 10.1371/journal.ppat.1001035

Holliday, R. (1974). "Ustilago maydis," in Handbook of Genetics, ed. R. C. King (New York, NY: Plenum Press), 575-595.

Hu, P., Zhang, Y. F., Cui, H. F., Yu, X. P., and Ye, Z. H. (2015). Cloning and expression analysis of transcription factor Rbf1 from Ustilago esculenta. J. Changjiang Vegetables 396, 206-209. doi: 10.3865/j.issn.1001-3547.2015.22. 072

Jiang, C., Zhang, X., Liu, H. Q., and Xu, J. R. (2018). Mitogen-activated protein kinase signaling in plant pathogenic fungi. PLos Pathog. 14:e1006875. doi: 10. 1371/journal.ppat.1006875

Jiang, M. X., Zhai, L. J., Yang, H., Zhai, S. M., and Zhai, C. K. (2016). Analysis of active components and proteomics of chinese wild rice (Zizania latifolia (Griseb) Turcz) and Indica rice (Nagina22). J. Med. Food 19, 798-804. doi: 10.1089/jmf.2015.3612

Jose, R. C., Goyari, S., Louis, B., Waikhom, S. D., Handique, P. J., and Talukdar, N. C. (2016). Investigation on the biotrophic interaction of Ustilago esculenta on Zizania latifolia found in the Indo-Burma biodiversity hotspot. Microb. Pathog. 98, 6-15. doi: 10.1016/j.micpath.2016.06.021

Jung, K. W., Kim, S. Y., Okagaki, L. H., Nielsen, K., and Bahn, Y. S. (2011). Ste50 adaptor protein governs sexual differentiation of Cryptococcus neoformans via the pheromone response MAPK signaling pathway. Fungal Genet. Biol. 48, 154-165. doi: 10.1016/j.fgb.2010.10.006

Kaffarnik, F., Muller, P., Leibundgut, M., Kahmann, R., and Feldbrugge, M. (2003). PKA and MAPK phosphorylation of Prf1 allows promoter discrimination in Ustilago maydis. EMBO J. 22, 5817-5826. doi: 10.1093/emboj/ cdg554

Kämper, J., Kahmann, R., Bölker, M., Ma, L. J., Brefort, T., Saville, B. J., et al. (2006). Insights from the genome of the biotrophic fungal plant pathogen Ustilago maydis. Nature 444, 97-101. doi: 10.1038/nature05248

Klosterman, S. J., Perlin, M. H., Garcia-Pedrajas, M., Covert, S. F., and Gold, S. E. (2007). Genetics of Morphogenesis and Pathogenic Development of Ustilago maydis. Adv. Genet. 57, 1-47. doi: 10.1016/S0065-2660(06)57001-4

Liang, S. W., Huang, Y. H., Chiu, J. Y., Tseng, H. W., Huang, J. H., and Shen, W. C. (2019). The smut fungus Ustilago esculenta has a bipolar mating system with three idiomorphs larger than 500kb. Fungal Genet. Biol. 126, 61-74. doi: 10.1016/j.fgb.2019.02.007

Maerz, S., and Seiler, S. (2010). Tales of RAM and MOR: NDR kinase signaling in fungal morphogenesis. Curr. Opin. Microbiol. 13, 663-671. doi: 10.1016/j.mib. 2010.08.010
Maidan, M. M., De Rop, L., Serneels, J., Exler, S., Rupp, S., Tournu, H., et al. (2005). The G protein-coupled receptor Gpr1 and the Galpha protein Gpa2 act through the cAMP-protein kinase A pathway to induce morphogenesis in Candida albicans. Mol. Biol. Cell 16, 1971-1986. doi: 10.1091/mbc.E04-09-0780

Martinez-Espinoza, A. D., Ruiz-Herrera, J., Leon-Ramirez, C. G., and Gold, S. E. (2004). MAP kinase and cAMP signaling pathways modulate the $\mathrm{pH}$-induced yeast-to-mycelium dimorphic transition in the corn smut fungus Ustilago maydis. Curr. Microbiol. 49, 274-281. doi: 10.1007/s00284-004-4315-6

Meng, X. Z., and Zhang, S. Q. (2013). MAPK cascades in plant disease resistance signaling. Annu. Rev. Phytopathol. 51, 245-266.

Müller, P., Aichinger, C., Feldbrügge, M., and Kahmann, R. (2000). The MAP kinase Kpp2 regulates mating and pathogenic development in Ustilago maydis. Mol. Microbiol. 34, 1007-1017. doi: 10.1046/j.1365-2958.1999.01661.x

Muller, P., Weinzierl, G., Brachmann, A., Feldbrugge, M., and Kahmann, R. (2003). Mating and pathogenic development of the Smut fungus Ustilago maydis are regulated by one mitogen-activated protein kinase cascade. Eukaryotic Cell 2, 1187-1199. doi: 10.1128/ec.2.6.1187-1199.2003

Raudaskoski, M., and Kothe, E. (2010). Basidiomycete mating type genes and pheromone signaling. Eukaryotic Cell 9, 847-859. doi: 10.1128/ec.00319-09

Russell, D. W., and Sambrook, J. (2001). Molecular Cloning: A Laboratory Manual. Cold Spring Harbour, NY: Harbor Laboratory Press,.

Saito, H. (2010). Regulation of cross-talk in yeast MAPK signaling pathways. Curr. Opin. Microbiol. 13, 677-683. doi: 10.1016/j.mib.2010.09.001

Sakulkoo, W., Oses-Ruiz, M., Garcia, E. O., Soanes, D. M., Littlejohn, G. R., Hacker, C., et al. (2018). A single fungal MAP kinase controls plant cell-to-cell invasion by the rice blast fungus. Science 359:1399. doi: 10.1126/science.aaq0892

Sartorel, E., and Perez-Martin, J. (2012). The distinct interaction between cell cycle regulation and the widely conserved morphogenesis-related (MOR) pathway in the fungus Ustilago maydis determines morphology. J. Cell Sci. 125, 4597-4608. doi: $10.1242 /$ jcs. 107862

Skalhegg, B. S., and Tasken, K. (2000). Specificity in the cAMP/PKA signaling pathway. Differential expression,regulation, and subcellular localization of subunits of PKA. Front. Biosci. J. Virtual Lib. 5:D678-D693. doi: 10.2741/ Skalhegg

Steinberg, G., Wedlich-Söldner, R., Brill, M., and Schulz, I. (2001). Microtubules in the fungal pathogen Ustilago maydis are highly dynamic and determine cell polarity. J. Cell Sci. 114(Pt 3), 609-622. doi: 10.1023/A:1005628808688

Suzuki, T., Choi, J. H., Kawaguchi, T., Yamashita, K., Morita, A., Hirai, H., et al. (2012). Makomotindoline from Makomotake, Zizania latifolia infected with Ustilago esculenta. Bioorg. Med. Chem. Lett. 22, 4246-4248. doi: 10.1016/j.bmcl. 2012.05.021

Szabo, Z., Tonnis, M., Kessler, H., and Feldbrugge, M. (2002). Structure-function analysis of lipopeptide pheromones from the plant pathogen Ustilago maydis. Mol. Genet. Genomics 268, 362-370. doi: 10.1007/s00438-002-0756-4

Verde, F., Wiley, D. J., and Nurse, P. (1998). Fission yeast orb6, a ser/thr protein kinase related to mammalian rho kinase and myotonic dystrophy kinase, is required for maintenance of cell polarity and coordinates cell morphogenesis with the cell cycle. Proc. Natl. Acad. Sci. U.S.A. 95, 7526-7531. doi: 10.1073/ pnas.95.13.7526

Vollmeister, E., Schipper, K., Baumann, S., Haag, C., Pohlmann, T., Stock, J., et al. (2012). Fungal development of the plant pathogen Ustilago maydis. Fems Microbiol Rev. 36, 59-77. doi: 10.1111/j.1574-6976.2011.00296.x

Wongsuk, T., Pumeesat, P., and Luplertlop, N. (2016). Fungal quorum sensing molecules: role in fungal morphogenesis and pathogenicity. J. Basic Microbiol. 56, 440-447. doi: 10.1002/jobm.201500759

Yan, M. X., Zhu, G. N., Lin, S., Xian, X. Y., Chang, C. Q., Xi, P. G., et al. (2016). The mating-type locus b of the sugarcane smut Sporisorium scitamineum is essential for mating, filamentous growth and pathogenicity. Fungal Genet. Biol. 86, 1-8. doi: 10.1016/j.fgb.2015.11.005

Yan, N., Du, Y. M., Liu, X. M., Chu, C., Shi, J., Zhang, H. B., et al. (2018). Morphological characteristics, nutrients, and bioactive compounds of Zizania latifolia, and health benefits of its seeds. Molecules 28:1561. doi: 10.3390/ molecules23071561

Ye, Z. H., Pan, Y., Zhang, Y. F., Cui, H. F., Jin, G. L., McHardy, A. C., et al. (2017). Comparative whole-genome analysis reveals artificial selection effects on Ustilago esculenta genome. DNA Res. 24, 635-648. doi: 10.1093/dnares/ dsx031 
Yu, J. J., Zhang, Y. F., Cui, H. F., Hu, P., Yu, X. P., and Ye, Z. H. (2015). An efficient genetic manipulation protocol for Ustilago esculenta. Fems Microbiol. Lett. 362:fnv087. doi: 10.1093/femsle/fnv087

Zarnack, K., Eichhorn, H., Kahmann, R., and Feldbruegge, M. (2008). Pheromoneregulated target genes respond differentially to MAPK phosphorylation of transcription factor Prf1. Mol. Microbiol. 69, 1041-1053. doi: 10.1111/j.13652958.2008.06345.x

Zhang, J. Z., Chu, F. Q., Guo, D. P., Hyde, K. D., and Xie, G. L. (2012). Cytology and ultrastructure of interactions between Ustilago esculenta and Zizania latifolia. Mycol. Prog. 11, 499-508. doi: 10.1007/s11557-011-0765-y

Zhang, Y. F., Cao, Q. C., Hu, P., Cui, H. F., Yu, X. P., and Ye, Z. H. (2017). Investigation on the differentiation of two Ustilago esculenta strains implications of a relationship with the host phenotypes appearing in the fields. BMC Microbiol. 17:228. doi: 10.1186/s12866-017-1138-8

Zhang, Y. F., Ge, Q. W., Cao, Q. C., Cui, H. F., Hu, P., Yu, X. P., et al. (2018a). Cloning and characterization of Two MAPK Genes UeKpp2 and UeKpp6 in Ustilago esculenta. Curr. Microbiol. 75, 1016-1024. doi: 10.1007/s00284-0181483-3

Zhang, Y. F., Liu, H. L., Cao, Q. C., Ge, Q. W., Cui, H. F., Yu, X. P., et al. (2018b). Cloning and characterization of UePrf1 gene in Ustilago esculenta. FEMS Microbiol. Lett. 365:fny081. doi: 10.1093/femsle/fny081

Zhang, Y. F., Yin, Y. M., Hu, P., Yu, J. J., Xia, W. Q., Ge, Q. W., et al. (2019). Mating-type loci of Ustilago esculenta are essential for mating and development. Fungal Genet. Biol. 125, 60-70. doi: 10.1016/j.fgb.2019. 01.008

Zhao, X., Kim, Y., Park, G., and Xu, J.-R. (2005). A mitogen-activated protein kinase cascade regulating infection-related morphogenesis in Magnaporthe grisea. Plant Cell 17, 1317-1329. doi: 10.1105/tpc.104.029116

Zhao, X., Mehrabi, R., and Xu, J. R. (2007). Mitogen-activated protein kinase pathways and fungal pathogenesis. Eukaryotic Cell 6, 1701-1714. doi: 10.1128/ EC.00216-07

Zuo, W. L., Oekmen, B., Depotter, J. R. L., Ebert, M. K., Redkar, A., Villamil, J. M., et al. (2019). "Molecular interactions between smut fungi and their host plants," in Annual Review of Phytopathology, eds J. E. Leach and S. E. Lindow (Palo Alto: ANNUAL REVIEWS), 411-430.

Conflict of Interest: The authors declare that the research was conducted in the absence of any commercial or financial relationships that could be construed as a potential conflict of interest.

Copyright (C) 2020 Zhang, Hu, Cao, Yin, Xia, Cui, Yu and Ye. This is an open-access article distributed under the terms of the Creative Commons Attribution License (CC BY). The use, distribution or reproduction in other forums is permitted, provided the original author(s) and the copyright owner(s) are credited and that the original publication in this journal is cited, in accordance with accepted academic practice. No use, distribution or reproduction is permitted which does not comply with these terms. 